\title{
CLASSIFICATION OF INDEFINITE HYPER-KÄHLER SYMMETRIC SPACES*
}

\author{
DMITRI V. ALEKSEEVSKY ${ }^{\dagger}$ AND VICENTE CORTÉS ${ }^{\ddagger}$
}

\begin{abstract}
We classify indefinite simply connected hyper-Kähler symmetric spaces. Any such space without flat factor has commutative holonomy group and signature $(4 m, 4 m)$. We establish a natural 1-1 correspondence between simply connected hyper-Kähler symmetric spaces of dimension $8 m$ and orbits of the group $\mathrm{GL}(m, \mathbb{H})$ on the space $\left(S^{4} \mathbb{C}^{n}\right)^{\tau}$ of homogeneous quartic polynomials $S$ in $n=2 m$ complex variables satisfying the reality condition $S=\tau S$, where $\tau$ is the real structure induced by the quaternionic structure of $\mathbb{C}^{2 m}=\mathbb{H}^{m}$. We define and classify also complex hyperKähler symmetric spaces. Such spaces without flat factor exist in any (complex) dimension divisible by 4 .
\end{abstract}

1. Introduction. We recall that a pseudo-Riemannian manifold $(M, g)$ is called a symmetric space if any point $x \in M$ is an isolated fixed point of an involutive isometry $s_{x}$ (called central symmetry with centre $x$ ). Since the product of two central symmetries $s_{x}$ and $s_{y}$ with sufficiently close centres is a shift along the geodesic $(x y)$, the group generated by central symmetries acts transitively on $M$ and one can identify $M$ with the quotient $M=G / K$, where $G$ is the connected component of the isometry group $\operatorname{Isom}(M, g)$ and $K$ is the stabilizer of a point $o \in M$.

A symmetric space $(M=G / K, g)$ is called Kähler (respectively, hyper-Kähler) if its holonomy group $\operatorname{Hol}(M, g)$ is a subgroup of the pseudo-unitary group $\mathrm{U}(p, q)$ (respectively, of the pseudo-symplectic group $\mathrm{Sp}(p, q) \subset \mathrm{SU}(2 p, 2 q)$ ). Any hyperKähler symmetric space is in particular a homogeneous hypercomplex manifold. Homogeneous hypercomplex manifolds of compact Lie groups were constructed by $\mathrm{Ph}$. Spindel, A. Sevrin, W. Troost, A. Van Proeyen [SSTVP] and by D. Joyce [J] and homogeneous hypercomplex structures on solvable Lie groups by M.L. Barberis and I. Dotti-Miatello [BD].

The classification of simply connected symmetric spaces reduces to the classification of involutive automorphisms $\sigma$ of a Lie algebra $\mathfrak{g}$, such that the adjoint representation $\operatorname{ad}_{\mathfrak{k}} \mid \mathfrak{m}$ preserves a pseudo-Euclidean scalar product $g$, where

$$
\mathfrak{g}=\mathfrak{k}+\mathfrak{m}, \quad \sigma|\mathfrak{k}=1, \quad \sigma| \mathfrak{m}=-1,
$$

is the eigenspace decomposition of the involution $\sigma$. Note that the eigenspace decomposition of an involutive automorphism is characterized by the conditions

$$
[\mathfrak{k}, \mathfrak{k}] \subset \mathfrak{k}, \quad[\mathfrak{k}, \mathfrak{m}] \subset \mathfrak{m}, \quad[\mathfrak{m}, \mathfrak{m}] \subset \mathfrak{k} .
$$

Such a decomposition is called a symmetric decomposition.

In fact, for any pseudo-Riemannian symmetric space $M=G / K+$ e conjugation with respect to the central symmetry $s_{o}$ with centre $o=e K$ is $a$. involutive automorphism of the Lie group $G$, which induces an involutive autom' phism $\sigma$ of its Lie algebra $\mathfrak{g}$. The pseudo-Riemannian metric of $M$ induces a $\mathfrak{k}$-invariant scalar product on $\mathfrak{m} \cong T_{o} M$, where $\mathfrak{g}=\mathfrak{k}+\mathfrak{m}$ is the symmetric decomposition defined by

\footnotetext{
${ }^{*}$ Received January 27, 2001; accepted for publication February 8, 2001.

${ }^{\dagger}$ Sophus Lie Center, Gen. Antonova 2 - 99, 117279 Moscow, and Department of Mathematics, University of Hull, Cottingham Road, Hull, HU6 7RX, UK (D.V.Alekseevsky@maths.hull.ac.uk).

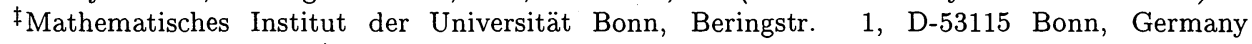
(vicente@math.uni-bonn.de).
} 
$\sigma$. Conversely, a symmetric decomposition $\mathfrak{g}=\mathfrak{k}+\mathfrak{m}$ together with a $\mathfrak{k}$-invariant scalar product on $\mathfrak{m}$ determines a pseudo-Riemannian symmetric space $M=G / K$, where $G$ is the simply connected Lie group with the Lie algebra $\mathfrak{g}, K$ is the connected (and closed) subgroup of $G$ generated by $\mathfrak{k}$, the pseudo-Riemannian metric on $M$ is defined by $g$ and the central symmetry is defined by the involutive automorphism $\sigma$ associated to the symmetric decomposition.

Naturally identifying the space $\mathfrak{m}$ with the tangent space $T_{o} M$, the isotropy group is identified with $\operatorname{Ad}_{K} \mid \mathfrak{m}$ and the holonomy algebra is identified with $\operatorname{ad}_{\mathfrak{h}}$, where $\mathfrak{h}=[\mathfrak{m}, \mathfrak{m}]$. If one assumes that the holonomy algebra is irreducible then one can prove that the Lie algebra $\mathfrak{g}$ is semisimple. Hence the classification of pseudoRiemannian symmetric spaces with irreducible holonomy reduces to the classification of involutive authomorphisms of semisimple Lie algebras. Such a classification was obtained by M. Berger [B1, B2] and A. Fedenko [F]. It includes the classification of Riemannian symmetric spaces (obtained earlier by E. Cartan), since according to de Rham's theorem any simply connected complete Riemannian manifold is a direct product of Riemannian manifolds with irreducible holonomy algebra and a Euclidean space.

A classification of pseudo-Riemannian symmetric spaces with non completely reducible holonomy is known only for signature $(1, n)$ (Cahen-Wallach [CW]) and for signature $(2, n)$ under the assumption that the holonomy group is solvable (Cahen-Parker $[\mathrm{CP}]$ ). The classification problem for arbitrary signature looks very complicated and includes, for example, the classification of Lie algebras which admit a nondegenerate ad-invariant symmetric bilinear form. An inductive construction of solvable Lie algebras with such a form was given by V. Kac [K], see also [MR1], [Bo] and [MR2].

In this paper we give a classification of pseudo-Riemannian hyper-Kähler symmetric spaces. In particular, we prove that any simply connected hyper-Kähler symmetric space $M$ has signature $(4 \mathrm{~m}, 4 \mathrm{~m})$ and its holonomy group is commutative. The main result is the following, see Theorem 6 .

Let $(E, \omega, j)$ be a complex symplectic vector space of dimension $4 m$ with a quaternionic structure $j$ such that $\omega(j x, j y)=\overline{\omega(x, y)}$ for all $x, y \in E$ and $E=E_{+} \oplus E_{-}$ a $j$-invariant Lagrangian decomposition. Such a decomposition exists if and only if the Hermitian form $\gamma=\omega(\cdot, j \cdot)$ has real signature $(4 m, 4 m)$. We denote by $\tau$ the real structure in $S^{2 r} E$ defined by $\tau\left(e_{1} e_{2} \ldots e_{2 r}\right):=j\left(e_{1}\right) j\left(e_{2}\right) \ldots j\left(e_{2 r}\right), e_{i} \in E$. Then any element $S \in\left(S^{4} E_{+}\right)^{\tau}$ defines a hyper-Kähler symmetric space $M_{S}$ which is associated with the symmetric decomposition

$$
\mathfrak{g}=\mathfrak{h}+\mathfrak{m}
$$

where $\mathfrak{m}=\left(\mathbb{C}^{2} \otimes E\right)^{\rho}$ is the fixed point set of the real structure $\rho$ on $\mathbb{C}^{2} \otimes E$ given by $\rho(h \otimes e)=j_{H} h \otimes j e$, where $j_{H}$ is the standard quaternionic structure on $\mathbb{C}^{2}=\mathbb{H}, \mathfrak{h}=$ $\operatorname{span}\left\{S_{e, e^{\prime}} \mid e, e^{\prime} \in E\right\}^{\tau} \subset \operatorname{sp}(E)^{\tau} \cong \operatorname{sp}(m, m)$ with the natural action on $\mathfrak{m} \subset \mathbb{C}^{2} \otimes E$ and the Lie bracket $\mathfrak{m} \wedge \mathfrak{m} \rightarrow \mathfrak{h}$ is given by

$$
\left[h \otimes e, h^{\prime} \otimes e^{\prime}\right]=\omega_{H}\left(h, h^{\prime}\right) S_{e, e^{\prime}},
$$

where $\omega_{H}$ is the standard complex symplectic form on $\mathbb{C}^{2}$.

Moreover, we establish a natural 1-1 correspondence between simply connected hyper-Kähler symmetric spaces (up to isomorphism) and orbits of the group $G L(m, \mathbb{H})$ in $\left(S^{4} E_{+}\right)^{\tau}$.

We define also the notion of complex hyper-Kähler symmetric space as a complex manifold $(M, g)$ of complex dimension $4 n$ with holomorphic metric $g$ such that for any 
point $x \in M$ there is a holomorphic central symmetry $s_{x}$ with centre $x$ and which has holonomy group $\operatorname{Hol}(M, g) \subset \operatorname{Sp}(n, \mathbb{C})(\operatorname{Sp}(n, \mathbb{C}) \hookrightarrow \operatorname{Sp}(n, \mathbb{C}) \times \operatorname{Sp}(n, \mathbb{C}) \subset \mathrm{O}(4 n, \mathbb{C})$ is diagonally embedded) and give a classification of such spaces. We establish a natural 1-1 correspondence between simply connected complex hyper-Kähler symmetric spaces and homogeneous polynomials of degree 4 in the vector space $\mathbb{C}^{n}$ considered up to linear transformations from $G L(n, \mathbb{C})$.

\section{Symmetric Spaces.}

2.1. Basic facts about pseudo-Riemannian symmetric spaces. A pseudo-Riemannian symmetric space is a pseudo-Riemannian manifold $(M, g)$ such that any point is an isolated fixed point of an isometric involution. Such a pseudo-Riemannian manifold admits a transitive Lie group of isometries $L$ and can be identified with $L / L_{o}$, where $L_{o}$ is the stabilizer of a point $o$. More precisely, any simply connected pseudo-Riemannian symmetric space $M=G / K$ is associated with a symmetric decomposition

$$
\mathfrak{g}=\mathfrak{k}+\mathfrak{m}, \quad[\mathfrak{k}, \mathfrak{k}] \subset \mathfrak{k}, \quad[\mathfrak{k}, \mathfrak{m}] \subset \mathfrak{m}, \quad[\mathfrak{m}, \mathfrak{m}] \subset \mathfrak{k}
$$

of the Lie algebra $\mathfrak{g}=\operatorname{Lie} G$ together with an $\mathrm{Ad}_{K}$-invariant pseudo-Euclidean scalar product on $\mathfrak{m}$. We will assume that $\mathrm{G}$ acts almost effectively on $M$, i.e. $\mathfrak{k}$ does not contain any nontrivial ideal of $\mathfrak{g}$, that $M$ and $G$ are simply connected and that $K$ is connected. Then, under the natural identification of the tangent space $T_{o} M$ at the canonical base point $o=e K$ with $\mathfrak{m}$, the holonomy group $\operatorname{Hol} \subset \operatorname{Ad}_{K} \mid \mathfrak{m}$. We will denote by $\mathfrak{h}$ the holonomy Lie algebra. Since the isotropy representation is faithful it is identified with the subalgebra $\mathfrak{h}=[\mathfrak{m}, \mathfrak{m}]:=\operatorname{span}\{[x, y] \mid x, y \in \mathfrak{m}\} \subset \mathfrak{k}$. Recall that the curvature tensor $R$ of a symmetric space $M$ at $o$ is $\mathfrak{h}$-invariant and determines the Lie bracket in the ideal $\mathfrak{h}+\mathfrak{m} \subset \mathfrak{g}$ as follows:

$$
\mathfrak{h}=R(\mathfrak{m}, \mathfrak{m}):=\operatorname{span}\{R(x, y) \mid x, y \in \mathfrak{m}\} \quad \text { and } \quad[x, y]=-R(x, y), \quad x, y \in \mathfrak{m} .
$$

The following result is well known:

Proposition 1. The full Lie algebra of Killing fields of a symmetric space has the form

$$
\operatorname{isom}(M)=\tilde{\mathfrak{h}}+\mathfrak{m}
$$

where the full isotropy subalgebra is given by

$$
\tilde{\mathfrak{h}}=\operatorname{aut}(R)=\{A \in \operatorname{so}(\mathfrak{m}) \mid A \cdot R=[A, R(\cdot, \cdot)]-R(A \cdot, \cdot)-R(\cdot, A \cdot)=0\} .
$$

2.2. Symmetric spaces of semisimple Lie groups. We will prove that in the case when $(M=G / K, g)$ is a pseudo-Riemannian symmetric space of a (connected) semisimple Lie group $G$ then $G$ is the maximal connected Lie group of isometries of $M$.

Proposition 2. Let $(M=G / K, g)$ be a pseudo-Riemannian symmetric space associated with a symmetric decomposition $\mathfrak{g}=\mathfrak{k}+\mathfrak{m}$. If $G$ is semisimple and almost effective then

(i) the restriction of the Cartan-Killing form $B$ of $\mathfrak{g}$ to $\mathfrak{k}$ is nondegenerate and hence $\mathfrak{k}$ is a reductive subalgebra of $\mathfrak{g}$ and $\mathfrak{g}=\mathfrak{k}+\mathfrak{m}$ is a B-orthogonal decomposition, 
(ii) $\mathfrak{k}=[\mathfrak{m}, \mathfrak{m}]$ and

(iii) $\mathfrak{g}=\operatorname{isom}(M, g)$ is the Lie algebra of the full isometry group of $M$.

Proof: For (i) see [O-V] Ch. 3 Proposition 3.6.

(ii) It is clear that $\overline{\mathfrak{g}}=[\mathfrak{m}, \mathfrak{m}]+\mathfrak{m}$ is an ideal of $\mathfrak{g}$. The $B$-orthogonal complement $\mathfrak{a}:=\overline{\mathfrak{g}}^{\perp} \subset \mathfrak{k}$ is a complementary ideal of $\mathfrak{g}$. Since $[\mathfrak{a}, \mathfrak{m}]=0$ the Lie algebra $\mathfrak{a}$ acts trivially on $M$. From the effectivity of $\mathfrak{g}$ we conclude that $\mathfrak{a}=0$.

(iii) By Proposition $1, \tilde{\mathfrak{g}}=\operatorname{isom}(M, g)=\tilde{\mathfrak{h}}+\mathfrak{m}$, where $\tilde{\mathfrak{h}}=\operatorname{aut}(R)=\{A \in \operatorname{so}(\mathfrak{m}) \mid$ $A \cdot R=0\}$. Now $\tilde{\mathfrak{h}}$ preserves $\mathfrak{m}$ and by the identity $A \cdot R=[A, R(\cdot, \cdot)]-R(A \cdot, \cdot)-R(\cdot, A \cdot)$ it also normalizes $\mathfrak{k}$. This shows that $\tilde{\mathfrak{h}}$ normalizes $\mathfrak{g}$ and hence $\mathfrak{g} \subset \tilde{\mathfrak{g}}$ is an ideal. Since $\mathfrak{g}$ is semisimple there exists a $\mathfrak{g}$-invariant complement $\mathfrak{b}$ in $\tilde{\mathfrak{g}}$. Note that $[\mathfrak{g}, \mathfrak{b}] \subset$ $\mathfrak{g} \cap \mathfrak{b}=0$. We can decompose any $X \in \mathfrak{b}$ as $X=Y+Z$, where $Y \in \tilde{\mathfrak{h}}$ and $Z \in \mathfrak{m}$. From $[\mathfrak{g}, \mathfrak{b}]=0$ it follows that $[\mathfrak{g}, Y]=[\mathfrak{g}, Z]=0$ and in particular $[\mathfrak{m}, Y]=0$. This implies that $Y=0$ and $X=Z \in \mathfrak{b} \cap \mathfrak{m}=0$. This shows that $\mathfrak{b}=0$ proving (iii).

We recall that a pseudo-Riemannian Hermitian symmetric space is pseudoRiemannian symmetric space $(M=G / K, g)$ together with an invariant (and hence parallel) $g$-orthogonal complex structure $J$.

Proposition 3. Let $(M=G / K, g, J)$ be a pseudo-Riemannian Hermitian symmetric space of a semisimple and almost effective Lie group $G$. Then the Ricci curvature of $M$ is not zero.

Proof: From Proposition 2 it follows that $\mathfrak{g}=\operatorname{isom}(M, g)=\tilde{\mathfrak{h}}+m$, where $\tilde{\mathfrak{h}}=\mathfrak{k}=$ $[\mathfrak{m}, \mathfrak{m}]$. It is well known that the curvature tensor $R$ of any pseudo-Kähler manifold (and in particular of any pseudo-Riemannian Hermitian symmetric space) is invariant under the operator $J$. This shows that $J \in \tilde{\mathfrak{h}}=\operatorname{aut}(R)=[\mathfrak{m}, \mathfrak{m}]=\mathfrak{h}$ (holonomy Lie algebra), which implies that the holonomy Lie algebra is not a subalgebra of $\mathrm{su}(\mathfrak{m}) \cong \operatorname{su}(p, q)$. Hence $M$ is not Ricci-flat. In fact, we can write $J=\sum \operatorname{ad}\left[X_{i}, Y_{i}\right]$, for $X_{i}, Y_{i} \in \mathfrak{m}$. Then using the formulas $-2 \operatorname{Ric}(X, J Y)=\operatorname{tr} J R(X, Y)$ for the Ricci curvature of a pseudo-Kähler manifold and $R(X, Y)=-a d_{[X, Y]} \mid \mathfrak{m}$ for the curvature of a symmetric space we calculate:

$$
-2 \sum \operatorname{Ric}\left(X_{i}, J Y_{i}\right)=\sum \operatorname{tr} J R\left(X_{i}, Y_{i}\right)=-\sum \operatorname{tr} J \operatorname{ad}\left[X_{i}, Y_{i}\right]=-\operatorname{tr} J^{2} \neq 0 .
$$

\section{Structure of Hyper-Kähler Symmetric Spaces.}

3.1. Definitions. A (possibly indefinite) hyper-Kähler manifold is a pseudoRiemannian manifold $\left(M^{4 n}, g\right)$ of signature $(4 k, 4 l)$ together with a compatible hypercomplex structure, i.e. three $g$-orthogonal parallel complex structures $\left(J_{1}, J_{2}, J_{3}=\right.$ $\left.J_{1} J_{2}\right)$. This means that the holonomy group Hol $\subset \operatorname{Sp}(k, l)$. Two hyper-Kähler manifolds $\left(M, g, J_{\alpha}\right)(\alpha=1,2,3)$ and $\left(M^{\prime}, g^{\prime}, J_{\alpha}^{\prime}\right)$ are called isomorphic if there exists a triholomorphic isometry $\varphi: M \rightarrow M^{\prime}$, i.e. $\varphi^{*} J_{\alpha}^{\prime}=J_{\alpha}$ and $\varphi^{*} g^{\prime}=g$.

A hyper-Kähler symmetric space is a pseudo-Riemannian symmetric space $(M=G / K, g)$ together with an invariant compatible hypercomplex structure. Consider now a simply connected hyper-Kähler symmetric space $\left(M=G / K, g, J_{\alpha}\right)$. Without restriction of generality we will assume that $G$ acts almost effectively. $M$ being hyper-Kähler is equivalent to $\operatorname{Ad}_{K} \mid \mathfrak{m} \subset \operatorname{Sp}(k, l)$, or, since $K$ is connected, to $\operatorname{ad}_{\mathfrak{k}} \mid \mathfrak{m} \subset \operatorname{sp}(k, l)$. This condition means that $\mathfrak{k}$ commutes with the Lie algebra $Q=\operatorname{sp}(1) \subset \operatorname{so}(\mathfrak{m})=\operatorname{so}(4 k, 4 l)$ spanned by three anticommuting complex structures $J_{1}, J_{2}, J_{3}$. 
3.2. Existence of a transitive solvable group of isometries and solvability of the holonomy. In this subsection we prove that any simply connected hyper-Kähler symmetric space $\left(M, g, J_{\alpha}\right)$ admits a transitive solvable Lie group $G \subset \operatorname{Aut}\left(g, J_{\alpha}\right)$ of automorphisms and has solvable holonomy group.

Proposition 4. Let $\left(M=G / K, g, J_{\alpha}\right)$ be a simply connected hyper-Kähler symmetric space and $A=\operatorname{Aut}_{0}(g, Q) \supset \operatorname{Aut}_{0}\left(g, J_{\alpha}\right) \supset G$ the connected group of isometries which preserve the quaternionic structure $Q=\operatorname{span}\left\{J_{\alpha}\right\}$. Then

(i) the stabilizer $A_{o}$ of a point $o \in M$ contains a maximal semisimple subgroup of $A$,

(ii) the radical $R$ of $A$ acts transitively and triholomorphically on $M$ and

(iii) the holonomy group of $M$ is solvable.

Proof: We consider the quaternionic Kähler symmetric space $\left(M=A / A_{o}, g, Q\right)$. The Lie algebra $\mathfrak{a}_{o}$ of the stabilizer is given by

$$
\mathfrak{a}_{o}=\operatorname{aut}(R, Q)=\left\{A \in \operatorname{so}\left(T_{o} M\right) \mid A \cdot R=0,[A, Q] \subset Q\right\} .
$$

Since the curvature tensor of a quaternionic Kähler manifold is invariant under the quaternionic structure $Q$ we conclude that $Q \subset \mathfrak{a}_{o}$ and $\mathfrak{a}_{o}=Q \oplus Z_{\mathfrak{a}}(Q)$, where $Z_{\mathfrak{a}}(Q)$ denotes the centralizer of $Q$ in $\mathfrak{a}$. Since $Q \cong \operatorname{sp}(1)$ is simple, we may choose a Levi-Malcev decomposition $\mathfrak{a}=\mathfrak{s}+\mathfrak{r}$ such that the Levi subalgebra $\mathfrak{s} \supset Q$. We put $\mathfrak{m}_{r}:=[Q, \mathfrak{r}]$ and denote by $\mathfrak{m}_{s}$ a $Q \oplus Z_{\mathfrak{s}}(Q)$-invariant complement of $Q$ in $[Q, \mathfrak{s}]$. The stabilizer has the decomposition $\mathfrak{a}_{o}=Q \oplus\left(Z_{\mathfrak{s}}(Q)+Z_{\mathfrak{r}}(Q)\right)$.

Lemma 1. The complement $\mathfrak{m}=\mathfrak{m}_{s}+\mathfrak{m}_{r}$ to $\mathfrak{a}_{o}$ in $\mathfrak{a}$ is $\mathfrak{a}_{o}$-invariant and the decomposition

$$
\mathfrak{a}=\mathfrak{a}_{o}+\mathfrak{m}
$$

is a symmetric decomposition.

Proof: It is clear that $\mathfrak{m}_{r}$ is $\mathfrak{a}_{o}$-invariant and $\mathfrak{m}_{s}$ is invariant under $Q \oplus Z_{\mathfrak{s}}(Q)$ by construction. It remains to check that $\left[Z_{\mathfrak{r}}(Q), \mathfrak{m}_{s}\right] \subset \mathfrak{m}$. Since $\mathfrak{m}_{s}=\left[Q, \mathfrak{m}_{s}\right]$, we have

$$
\left[Z_{\mathfrak{r}}(Q), \mathfrak{m}_{s}\right]=\left[Z_{\mathfrak{r}}(Q),\left[Q, \mathfrak{m}_{s}\right]\right]=\left[Q,\left[Z_{\mathfrak{r}}(Q), \mathfrak{m}_{s}\right]\right] \subset[Q, \mathfrak{r}]=\mathfrak{m}_{r} \subset \mathfrak{m}
$$

This shows that $\mathfrak{a}=\mathfrak{a}_{o}+\mathfrak{m}$ is an $\mathfrak{a}_{o}$-invariant decomposition. We denote by $\mathfrak{a}=\mathfrak{a}_{o}+\mathfrak{p}$ a symmetric decomposition. Any other $\mathfrak{a}_{o}$-invariant decomposition is of the form $\mathfrak{a}=\mathfrak{a}_{o}+\mathfrak{p}_{\varphi}$, where $\varphi: \mathfrak{p} \rightarrow \mathfrak{a}_{o}$ is an $\mathfrak{a}_{o}$-equivariant map and $\mathfrak{p}_{\varphi}=\{X+\varphi(X) \mid X \in \mathfrak{p}\}$ If such non-zero equivariant map $\varphi$ exists then $\mathfrak{p}$ and $\mathfrak{a}_{o}$ contain non-trivial isomorphic $Q$-submodules. Since $\mathfrak{p}$ is a sum of 4-dimensional irreducible $Q$-modules and $\mathfrak{a}_{o}$ is the sum of the 3-dimensional irreducible $Q$-module $Q$ and the trivial complementary $Q$-module $Z_{\mathfrak{a}_{o}}(Q)$, we infer that there exists a unique $\mathfrak{a}_{o}$-invariant decomposition, which coincides with the symmetric decomposition $\mathfrak{a}=\mathfrak{a}_{o}+\mathfrak{p}$.

To prove (i) we have to check that $\mathfrak{m}_{s}=0$. We note that by the previous lemma $\mathfrak{s}=\left(Q \oplus \mathfrak{Z}_{\mathfrak{s}}(Q)\right)+\mathfrak{m}_{s}$ is a symmetric decomposition of the semisimple Lie algebra $\mathfrak{s}$. Since $\left[\mathfrak{m}_{s}, \mathfrak{m}_{s}\right] \subset Z_{\mathfrak{s}}(Q)$ it defines a hyper-Kähler symmetric space $N=L / L_{o}$, where $L$ is the simply connected semisimple Lie group with Lie algebra $\mathfrak{l}=Z_{\mathfrak{s}}(Q)+\mathfrak{m}_{s}$ and $L_{o}$ is the Lie subgroup generated by the subalgebra $Z_{\mathfrak{s}}(Q) \subset \mathfrak{l}$. Since $N$ is in particular a Ricci-flat pseudo-Riemannian Hermitian symmetric space, from Proposition 3 we obtain that $N$ is reduced to point. Therefore $\mathfrak{m}_{s}=0$. This proves (i) and (ii). Finally, since the holonomy Lie algebra $\mathfrak{h}$ is identified with $\mathfrak{h}=[\mathfrak{m}, \mathfrak{m}]=\left[\mathfrak{m}_{r}, \mathfrak{m}_{r}\right] \subset \mathfrak{r}$ it is solvable as subalgebra of the solvable Lie algebra $\mathfrak{r}$. 
3.3. Hyper-Kähler symmetric spaces and second prolongation of symplectic Lie algebras. Let $\left(M=G / K, g, J_{\alpha}\right)$ be a simply connected hyper-Kähler symmetric space associated with a symmetric decomposition (2.1). Without restriction of generality we will assume that $G$ acts almost effectively and that $\mathfrak{k}=[\mathfrak{m}, \mathfrak{m}]=$ $\mathfrak{h}$ (holonomy Lie algebra). The complexification $\mathfrak{m}^{\mathbb{C}}$ as $\mathfrak{h}^{\mathbb{C}}$-module can be written as $\mathfrak{m}^{\mathbb{C}}=H \otimes E$, such that $\mathfrak{h}^{\mathbb{C}} \subset \mathrm{Id} \otimes \operatorname{sp}(E) \cong \operatorname{sp}(E)$, where $H=\mathbb{C}^{2}$ and $E=\mathbb{C}^{2 n}$ are complex symplectic vector spaces with symplectic form $\omega_{H}$ and $\omega_{E}$, respectively, such that $g^{\mathbb{C}}=\omega_{H} \otimes \omega_{E}$ is the complex bilinear metric on $\mathfrak{m}^{\mathbb{C}}$ induced by $g$. Note that the symplectic forms are unique up to the transformation $\omega_{H} \mapsto \lambda \omega_{H}$, $\omega_{E} \mapsto \lambda^{-1} \omega_{E}, \lambda \in \mathbb{C}^{*}$. We have also quaternionic structures $j_{H}$ and $j_{E}$ on $H$ and $E$, such that $\omega_{H}\left(j_{H} x, j_{H} y\right)=\overline{\omega_{H}(x, y)}$ for all $x, y \in H$ and $\omega_{E}\left(j_{E} x, j_{E} y\right)=\overline{\omega_{E}(x, y)}$ for all $x, y \in E$, where the bar denotes complex conjugation. This implies that $\gamma_{H}:=\omega_{H}\left(\cdot, j_{H} \cdot\right)$ and $\gamma_{E}:=\omega_{E}\left(\cdot, j_{E} \cdot\right)$ are Hermitian forms on $H$ and $E$. For fixed $\omega_{H}$ and $\omega_{E}$ the quaternionic structures $j_{H}$ and $j_{E}$ are uniquely determined if we require that $\gamma_{H}$ is positive definite and that $\rho=j_{H} \otimes j_{E}$ is the real structure on $\mathfrak{m}^{\mathbb{C}}$, i.e. the complex conjugation with respect to $\mathfrak{m}$. The metric $g^{\mathbb{C}}$ and the Hermitian form $g^{\mathbb{C}}(\cdot, \rho \cdot)=\gamma_{H} \otimes \gamma_{E}$ restrict to a real valued scalar product $g$ of some signature $(4 k, 4 l)$ on $\mathfrak{m}=(H \otimes E)^{\rho}$, where $(2 k, 2 l)$ is the (real) signature of the Hermitian form $\gamma_{E}=\omega_{E}\left(\cdot, j_{E} \cdot\right)$. Note that for the holonomy algebra we have the inclusion

$$
\begin{gathered}
\mathfrak{h}=\operatorname{Id} \otimes\left(\mathfrak{h}^{\mathbb{C}}\right)^{j_{E}} \hookrightarrow \operatorname{sp}(E)^{j_{E}}=\left\{A \in \operatorname{sp}(E) \mid\left[A, j_{E}\right]=0\right\} \\
=\operatorname{aut}\left(E, \omega_{E}, j_{E}\right) \cong \operatorname{aut}\left(\mathfrak{m}, g, J_{\alpha}\right) \cong \operatorname{sp}(k, l) .
\end{gathered}
$$

Using the symplectic forms we identify $H=H^{*}$ and $E=E^{*}$. Then the symplectic Lie algebras are identified with symmetric tensors as follows:

$$
\operatorname{sp}(H)=S^{2} H, \quad \operatorname{sp}(E)=S^{2} E .
$$

Since the curvature tensor $R$ of any hyper-Kähler manifold $M^{4 n}$ at a point $p \in M$ can be identified with an element $R \in S^{2} \mathrm{sp}(k, l)$ it is invariant under the Lie algebra $\operatorname{sp}(1)=\operatorname{span}\left\{J_{1}, J_{2}, J_{3}\right\}$. Let $M=G / K$ be a hyper-Kähler symmetric space as above. By Proposition 1 we can extend the Lie algebra $\mathfrak{g}=\mathfrak{k}+\mathfrak{m}=\mathfrak{h}+\mathfrak{m}$ to a Lie algebra

$$
\tilde{\mathfrak{g}}=\operatorname{sp}(1)+\mathfrak{h}+\mathfrak{m}
$$

of Killing vector fields such that $[\mathrm{sp}(1), \mathfrak{h}]=0$. In the $H \otimes E$-formalism the Lie algebra $\operatorname{sp}(1)$ is identified with $\operatorname{sp}(H)^{j_{H}} \otimes \operatorname{Id} \subset \operatorname{so}(\mathfrak{m})$.

Lemma 2. Denote by $\tilde{\mathfrak{g}}^{\mathbb{C}}=\operatorname{sp}(1, \mathbb{C})+\mathfrak{h}^{\mathbb{C}}+\mathfrak{m}^{\mathbb{C}}$ the complexification of the Lie algebra $\tilde{\mathfrak{g}}$. Then the Lie bracket $[\cdot, \cdot]: \wedge^{2} \mathfrak{m}^{\mathbb{C}} \rightarrow \mathfrak{h}^{\mathbb{C}}$ can be written as

$$
\left[h \otimes e, h^{\prime} \otimes e^{\prime}\right]=\omega_{H}\left(h, h^{\prime}\right) S_{e, e^{\prime}},
$$

where $S \in\left(\mathfrak{h}^{\mathbb{C}}\right)^{(2)}:=\mathfrak{h}^{\mathbb{C}} \otimes S^{2} E^{*} \cap E \otimes S^{3} E^{*}=\mathfrak{h}^{\mathbb{C}} \otimes \mathfrak{h}^{\mathbb{C}} \cap S^{4} E$. Moreover $S$ is $\operatorname{sp}(1, \mathbb{C}) \oplus$ $\mathfrak{h}^{\mathbb{C}}$-invariant and satisfies the following reality condition: $\left[S_{j_{E} e, e^{\prime}}-S_{e, j_{E} e^{\prime}}, j_{E}\right]=0$.

Proof: The Lie bracket $[\cdot, \cdot]: \wedge^{2} \mathfrak{m}^{\mathbb{C}} \rightarrow \mathfrak{h}^{\mathbb{C}}$ is an $\operatorname{sp}(1, \mathbb{C}) \oplus \mathfrak{h}^{\mathbb{C}}$-equivariant map, due to the Jacobi identity. We decompose the $\operatorname{sp}(H) \oplus \operatorname{sp}(E)$-module $\wedge^{2} \mathfrak{m}^{\mathbb{C}}$ :

$$
\wedge^{2} \mathfrak{m}^{\mathbb{C}}=\wedge^{2}(H \otimes E)=\wedge^{2} H \otimes S^{2} E \oplus S^{2} H \otimes \wedge^{2} E=\omega_{H} \otimes S^{2} E \oplus S^{2} H \otimes \wedge^{2} E .
$$


Since $\mathfrak{h}^{\mathbb{C}} \subset S^{2} E$ the Lie bracket defines an $\operatorname{sp}(1, \mathbb{C}) \oplus \mathfrak{h}^{\mathbb{C}}$-invariant element of the space $\omega_{H} \otimes S^{2} E \otimes S^{2} E \oplus S^{2} H \otimes \wedge^{2} E \otimes S^{2} E$. The second summand has no nontrivial $\operatorname{sp}(1, \mathbb{C})-$ invariant elements. Hence the bracket is of the form (3.1), where $S \in S^{2} E^{*} \otimes \mathfrak{h}^{\mathbb{C}} \subset$ $S^{2} E \otimes S^{2} E$. The Jacobi identity reads:

$$
\begin{aligned}
0= & {\left[h \otimes e,\left[h^{\prime} \otimes e^{\prime}, h^{\prime \prime} \otimes e^{\prime \prime}\right]\right]-\left[\left[h \otimes e, h^{\prime} \otimes e^{\prime}\right], h^{\prime \prime} \otimes e^{\prime \prime}\right]-\left[h^{\prime} \otimes e^{\prime},\left[h \otimes e, h^{\prime \prime} \otimes e^{\prime \prime}\right]\right] } \\
& =-\omega_{H}\left(h^{\prime}, h^{\prime \prime}\right) h \otimes S_{e^{\prime}, e^{\prime \prime}} e-\omega_{H}\left(h, h^{\prime}\right) h^{\prime \prime} \otimes S_{e, e^{\prime}} e^{\prime \prime}+\omega_{H}\left(h, h^{\prime \prime}\right) h^{\prime} \otimes S_{e, e^{\prime \prime}} e^{\prime}
\end{aligned}
$$

Since $\operatorname{dim} H=2$ we may assume that $h, h^{\prime}=h^{\prime \prime}$ is a symplectic basis, i.e. $\omega_{H}\left(h, h^{\prime}\right)=$ 1, and the equation implies: $S_{e, e^{\prime \prime}} e^{\prime}=S_{e, e^{\prime}} e^{\prime \prime}$, i.e. $S \in\left(\mathfrak{h}^{\mathbb{C}}\right)^{(2)}$. The Lie bracket of two real elements $h \otimes e+j_{H} h \otimes j_{E} e$ and $h \otimes e^{\prime}+j_{H} h \otimes j_{E} e^{\prime} \in \mathfrak{m} \subset \mathfrak{m}^{\mathbb{C}}$ is an element of $\mathfrak{h}$. This gives:

$$
\left[h \otimes e+j_{H} h \otimes j_{E} e, h \otimes e^{\prime}+j_{H} h \otimes j_{E} e^{\prime}\right]=\omega_{H}\left(h, j_{H} h\right)\left(S_{e, j_{E} e^{\prime}}-S_{j_{E} e, e^{\prime}}\right) \in \mathfrak{h} .
$$

From the fact that the Hermitian form $\gamma_{H}=\omega_{H}\left(\cdot, j_{H} \cdot\right)$ is positive definite it follows that $\omega_{H}\left(h, j_{H} h\right) \neq 0$. This establishes the reality condition since $\mathfrak{h}=\{A \in$ $\left.\mathfrak{h}^{\mathbb{C}} \mid\left[A, j_{E}\right]=0\right\}$.

In fact any tensor $S \in S^{4} E$ satisfying the conditions of the above lemma can be used to define a hyper-Kähler symmetric space as the following theorem shows. We can identify $S^{4} E$ with the space $\mathbb{C}[E]^{(4)}$ of homogeneous quartic polynomials on $E \cong E^{*}$.

THEOREM 1. Let $S \in S^{4} E, E=\mathbb{C}^{2 n}$, be a quartic polynomial invariant under all endomorphisms $S_{e, e^{\prime}} \in S^{2} E=\operatorname{sp}(E)$ and satisfying the reality condition

$$
\left[S_{j_{E} e e^{\prime}}-S_{e, j_{E} e^{\prime}}, j_{E}\right]=0
$$

Then it defines a hyper-Kähler symmetric space, which is associated with the following complex symmetric decomposition

$$
\mathfrak{g}^{\mathbb{C}}=\mathfrak{h}^{\mathbb{C}}+H \otimes E, \quad \mathfrak{h}^{\mathbb{C}}=\operatorname{span}\left\{S_{e, e^{\prime}} \mid e, e^{\prime} \in E\right\} \subset \operatorname{sp}(E) .
$$

The bracket $\wedge^{2}(H \otimes E) \rightarrow \mathfrak{h}^{\mathbb{C}}$ is given by (3.1). The real symmetric decomposition is defined as $\rho$-real form $\mathfrak{g}=\mathfrak{h}+\mathfrak{m}$ of (3.3), where

$$
\mathfrak{k}=\mathfrak{h}=\left\{A \in \mathfrak{h}^{\mathbb{C}} \mid\left[A, j_{E}\right]=0\right\}=\operatorname{span}\left\{S_{j_{E} e, e^{\prime}}-S_{e, j_{E} e^{\prime}} \mid e, e^{\prime} \in E\right\}, \quad \mathfrak{m}=(H \otimes E)^{\rho} .
$$

The hyper-Kähler symmetric space $M$ associated to this symmetric decomposition is the quotient $M=M_{S}=G / K$, where $G$ is the simply connected Lie group with Lie algebra $\mathfrak{g}$ and $K \subset G$ is the connected (and closed) subgroup with Lie algebra $\mathfrak{k}=\mathfrak{h}$.

Moreover any simply connected hyper-Kähler symmetric space can be obtained by this construction. Two hyper-Kähler symmetric spaces $M_{S}$ and $M_{S^{\prime}}$ defined by quartics $S$ and $S^{\prime}$ are isomorphic if and only if $S$ and $S^{\prime}$ are in the same orbit of the group $\operatorname{Aut}\left(E, \omega_{E}, j_{E}\right)=\left\{A \in \operatorname{Sp}(E) \mid\left[A, j_{E}\right]=0\right\} \cong \operatorname{Sp}(k, l)$.

Proof: First of all we note that $\mathfrak{h}^{\mathbb{C}}=S_{E, E}:=\operatorname{span}\left\{S_{e, e^{\prime}} \mid e, e^{\prime} \in E\right\}$ is a subalgebra of $\operatorname{sp}(E)$ because

$$
\left[S_{e, e^{\prime}}, S_{f, f^{\prime}}\right]=\left(S_{e, e^{\prime}} \cdot S\right)_{f, f^{\prime}}-S_{S_{e, e^{\prime}} f, f^{\prime}}-S_{f, S_{e, e^{\prime}} f^{\prime}}=-S_{S_{e, e^{\prime}} f, f^{\prime}}-S_{f, S_{e, e^{\prime}} f^{\prime}} \in \mathfrak{h}^{\mathbb{C}}
$$


Since $S$ is $\mathfrak{h}^{\mathbb{C}}$-invariant and completely symmetric we can check, as in Lemma 2, that the Jacobi identity is satisfied and that (3.3) defines a complex symmetric decomposition. We prove that $\mathfrak{h}:=\operatorname{span}\left\{S_{j_{E} e, e^{\prime}}-S_{e, j_{E} e^{\prime}} \mid e, e^{\prime} \in E\right\} \subset\left\{A \in \mathfrak{h}^{\mathbb{C}} \mid\left[A, j_{E}\right]=0\right\}$ defines a real form of $\mathfrak{h}^{\mathbb{C}}$. Indeed for $e, e^{\prime} \in E$ we have

$$
\begin{aligned}
& S_{e, e^{\prime}}=\frac{1}{2}\left(S_{e, e^{\prime}}+S_{j_{E} e, j_{E} e^{\prime}}\right)-\frac{\sqrt{-1}}{2}\left(\sqrt{-1} S_{e, e^{\prime}}-\sqrt{-1} S_{j_{E} e, j_{E} e^{\prime}}\right) \\
& =\frac{1}{2}\left(S_{j_{E} e^{\prime \prime}, e^{\prime}}-S_{e^{\prime \prime}, j_{E} e^{\prime}}\right)-\frac{\sqrt{-1}}{2}\left(S_{j_{E} e^{\prime \prime}, \sqrt{-1} e^{\prime}}-S_{e^{\prime \prime}, j_{E} \sqrt{-1} e^{\prime}}\right)
\end{aligned}
$$

where $e^{\prime \prime}=-j_{E} e$. Due to the reality condition the restriction of the Lie bracket $[\cdot, \cdot]: \wedge^{2} \mathfrak{m}^{\mathbb{C}} \rightarrow \mathfrak{h}^{\mathbb{C}}$ to $\wedge^{2} \mathfrak{m}$ has values in $\mathfrak{h}$ and $\mathfrak{g}=\mathfrak{h}+\mathfrak{m}$ is a symmetric decomposition with $[\mathfrak{m}, \mathfrak{m}]=\mathfrak{h}$. The metric $g^{\mathbb{C}}=\omega_{H} \otimes \omega_{E}$ defines a real valued scalar product $g$ of some signature $(p, q)$ on $\mathfrak{m}=(H \otimes E)^{\rho}$, which is invariant under the Lie algebra $\mathfrak{h}$. Since $\left[\mathfrak{h}, j_{E}\right]=0$ the holonomy algebra $\mathfrak{h} \subset \operatorname{sp}(k, l), p=4 k, q=4 l$. Hence this symmetric decomposition defines a hyper-Kähler symmetric space.

By L’emma 2 any hyper-Kähler symmetric space can be obtained by this construction. It is well known that a simply connected symmetric space $M$ of signature $(p, q)$ is determined by its abstract curvature tensor $R \in S^{2}\left(\wedge^{2} V\right), V=\mathbb{R}^{p, q}$, and two tensors $R$ and $R^{\prime}$ define isometric symmetric spaces if and only if they belong to the same $O(V)$ orbit. Similarly a simply connected hyper-Kähler symmetric space is determined up to isometry by its abstract curvature tensor $R \in S^{2}\left(\wedge^{2} V\right)$, where $V=\mathbb{R}^{4 k, 4 l}$ is the pseudo-Euclidean vector space with fixed hypercomplex structure $J_{\alpha} \in O(V)$. For a hyper-Kähler symmetric space the complexified curvature tensor has the form

$$
R\left(h \otimes e, h^{\prime} \otimes e^{\prime}\right)=-\omega_{H}\left(h, h^{\prime}\right) S_{e, e^{\prime}}
$$

where $S \in S^{4} E$ is the quartic form of Lemma 2. Two such curvature tensors define isomorphic hyper-Kähler symmetric spaces if and only if they belong to the same orbit of $\operatorname{Aut}\left(\mathbb{R}^{4 k, 4 l}, J_{\alpha}\right)=\operatorname{Sp}(k, l)$. The group $\operatorname{Sp}(k, l)$ acts on $V^{\mathbb{C}}=H \otimes E$ as $\mathrm{Id} \otimes \operatorname{Sp}(E)^{j_{E}}=\mathrm{Id} \otimes \operatorname{Aut}\left(E, \omega_{E}, j_{E}\right)$. Hence two curvature tensors $R=-\omega_{H} \otimes S$ and $R^{\prime}=-\omega_{H} \otimes S^{\prime}$ are in the same $\operatorname{Sp}(k, l)$-orbit if and only if $S$ and $S^{\prime}$ are in the same $\operatorname{Sp}(k, l)$-orbit on $S^{4} E$.

\section{Complex Hyper-Kähler Symmetric Spaces.}

4.1. Complex hyper-Kähler manifolds. A complex Riemannian manifold is a complex manifold $M$ equipped with a complex metric $g$, i.e. a holomorphic section $g \in \Gamma\left(S^{2} T^{*} M\right)$ which defines a nondegenerate complex quadratic form. As in the real case any such manifold has a unique holomorphic torsionfree and metric connection (Levi-Civita connection). A complex hyper-Kähler manifold is a complex Riemannian manifold $\left(M^{4 n}, g\right)$ of complex dimension $4 n$ together with a compatible hypercomplex structure, i.e. three $g$-orthogonal parallel complex linear endomorphisms $\left(J_{1}, J_{2}, J_{3}=J_{1} J_{2}\right)$ with $J_{\alpha}^{2}=-1$. This means that the holonomy group Hol $\subset \operatorname{Sp}(n, \mathbb{C})=Z_{O(4 n, \mathbb{C})}(\operatorname{Sp}(1, \mathbb{C}))$. The linear group $\operatorname{Sp}(n, \mathbb{C})$ is diagonally embedded into $\operatorname{Sp}(n, \mathbb{C}) \times \operatorname{Sp}(n, \mathbb{C}) \subset \mathrm{GL}(4 n, \mathbb{C})$. Two complex hyper-Kähler manifolds $\left(M, g, J_{\alpha}\right)(\alpha=1,2,3)$ and $\left(M^{\prime}, g^{\prime}, J_{\alpha}^{\prime}\right)$ are called isomorphic if there exists a holomorphic isometry $\varphi: M \rightarrow M^{\prime}$ such that $\varphi^{*} J_{\alpha}^{\prime}=J_{\alpha}$ and $\varphi^{*} g^{\prime}=g$. 
We will show that the complex hyper-Kähler structure can be described as a halfflat Grassmann structure of a certain type. A Grassmann structure on a complex Riemannian manifold $(M, g)$ is a decomposition of the (holomorphic) tangent bundle $T M \cong H \otimes E$ into the tensor product of two holomorphic vector bundles $H$ and $E$ of rank $2 m$ and $2 n$ with holomorphic nondegenerate 2-forms $\omega_{H}$ and $\omega_{E}$ such that $g=\omega_{H} \otimes \omega_{E}$. The Grassmann structure will be called parallel if the Levi-Civita connection $\nabla=\nabla^{T M}$ can be decomposed as:

$$
\nabla=\nabla^{H} \otimes \mathrm{Id}+\mathrm{Id} \otimes \nabla^{E}
$$

where $\nabla^{H}$ and $\nabla^{E}$ are (uniquely defined) symplectic connections in the bundles $H$ and $E$. A parallel Grassmann structure will be called half-flat if $\nabla^{H}$ is flat. Note that a parallel Grassmann structure on a simply connected manifold is half-flat if and only if the holonomy group of the Levi-Civita connection is contained in $\operatorname{Id} \otimes \operatorname{Sp}(n, \mathbb{C}) \subset$ $\mathrm{Sp}(m, \mathbb{C}) \otimes \operatorname{Sp}(n, \mathbb{C}) \subset \mathrm{O}\left(\mathbb{C}^{2 m} \otimes \mathbb{C}^{2 n}\right)$.

Proposition 5. A complex hyper-Kähler structure $\left(g, J_{\alpha}\right)$ on a simply connected complex manifold $M$ is equivalent to the following geometric data:

(i) a half-flat Grassmann structure $(T M, g, \nabla) \cong\left(H, \omega_{H}, \nabla^{H}\right) \otimes\left(E, \omega_{E}, \nabla^{E}\right)$ and

(ii) an isomorphism of flat symplectic vector bundles $H \cong M \times \mathbb{C}^{2}$. Under this isomorphism $\omega_{H}=h_{1}^{*} \wedge h_{2}^{*}$, where $\left(h_{1}, h_{2}\right)$ is the standard basis of $\mathbb{C}^{2}$ considered as parallel frame of the trivial bundle $H=M \times \mathbb{C}^{2}$.

More precisely,

$$
J_{1}=R_{i} \otimes \mathrm{Id}, \quad J_{2}=R_{j} \otimes \mathrm{Id}, \quad \text { and } \quad J_{3}=R_{k} \otimes \mathrm{Id},
$$

where we have identified $\mathbb{C}^{2}=\mathbb{C h}_{1} \oplus \mathbb{C} h_{2}$ with $\mathbb{H}=\operatorname{span}_{\mathbb{R}}\{1, i, j, k\}=\operatorname{span}_{\mathbb{C}}\{1, j\}=$ $\mathbb{C} 1 \oplus \mathbb{C} j$ with the complex structure defined by left-multiplication by $i$ and $R_{x}$ denotes the right-multiplication by the quaternion $x \in \mathbb{H}$.

Proof: It is easy to check that the geometric data (i) and (ii) define a complex hyper-Kähler structure on $M$. Conversely let $\left(g, J_{\alpha}\right)$ be a complex hyper-Kähler structure on $M$. The endomorphism $J_{1}$ has eigenvalues $\pm i$ and the tangent space can be decomposed into a sum of eigenspaces

$$
T M=E_{+} \oplus E_{-} .
$$

From the $J_{1}$-invariance of the metric $g$ it follows that $g\left(E_{ \pm}, E_{ \pm}\right)=0$ and we can identify $E_{-}=E^{*}$ with the dual space of $E=E_{+}$. Since $J_{2}$ anticommutes with $J_{1}$ it interchanges $E$ and $E^{*}$ and hence defines an isomorphism $E \stackrel{\sim}{\rightarrow} E^{*}$. Now $g\left(\cdot, J_{2} \cdot\right)$ defines a symplectic form $\omega_{E}$ on $E$. Let $H=M \times \mathbb{C}^{2}=M \times\left(\mathbb{C} h_{1} \oplus \mathbb{C} h_{2}\right)$ be the trivial bundle with 2-form $\omega_{H}=h_{1}^{*} \wedge h_{2}^{*}$. Then we can identify

$$
T M=E \oplus E^{*}=E \oplus E=h_{1} \otimes E \oplus h_{2} \otimes E=H \otimes E .
$$

We check that under this identification we have $g=\omega_{H} \otimes \omega_{E}$. Note that both sides vanish on $h_{1} \otimes E$ and $h_{2} \otimes E$ and $\omega_{H}\left(h_{1}, h_{2}\right)=1$. We calculate for $e, e^{\prime} \in E=E_{+}=$ $h_{1} \otimes E:$

$$
g\left(e, J_{2} e^{\prime}\right)=\omega_{E}\left(e, e^{\prime}\right)=\omega_{H}\left(h_{1}, h_{2}\right) \omega_{E}\left(e, e^{\prime}\right)=\left(\omega_{H} \otimes \omega_{E}\right)\left(h_{1} \otimes e, h_{2} \otimes e^{\prime}\right) .
$$

Hence we have a Grassmann structure. The eigenspaces $E_{ \pm}$of the parallel endomorphism $J_{1}$ are invariant under parallel transport. Therefore the Levi-Civita connection 
$\nabla$ induces a connection $\nabla^{E}$ in the bundle $E$. Since $\nabla g=0$ and $\nabla J_{2}=0$ we have $\nabla^{E} \omega_{E}=0$. We define a flat connection $\nabla^{H}$ on the trivial bundle $H=M \times \mathbb{C}^{2}$ by the condition $\nabla^{H} h_{1}=\nabla^{H} h_{2}=0$. Then $\nabla=\nabla^{H} \otimes I d+I d \otimes \nabla^{E}$. So the Grassmann structure is half-flat.

Finally, using the standard identification $\mathbb{C}^{2}=\mathbb{H}$, one can easily check that the $J_{\alpha}$ are given by (4.1).

4.2. Complexification of real hyper-Kähler manifolds. Let $\left(M, g, J_{\alpha}\right)$ be a (real) hyper-Kähler manifold. We will assume that it is real analytic. This is automatically true if the metric $g$ is positive definite since it is Ricci-flat and a fortiori Einstein. Using analytic continuation we can extend $\left(M, g, J_{\alpha}\right)$ to a complex hyperKähler manifold $\left(M^{\mathbb{C}}, g^{\mathbb{C}}, J_{\alpha}^{\mathbb{C}}\right)$ equipped with an antiholomorphic involution $T$. In complex local coordinates $z^{j}=x^{j}+i y^{j}$ which are extension of real analytic coordinates $x^{j}, y^{j}$ the involution is given by the complex conjugation $z^{j} \rightarrow \bar{z}^{j}=x^{j}-i y^{j}$. We can reconstruct the (real) hyper-Kähler manifold as the fixed point set of $T$. We will call $\left(M, g, J_{\alpha}\right)$ a real form of $\left(M^{\mathbb{C}}, g^{\mathbb{C}}, J_{\alpha}^{\mathbb{C}}\right)$ and $\left(M^{\mathbb{C}}, g^{\mathbb{C}}, J_{\alpha}^{\mathbb{C}}\right)$ the complexification of $\left(M, g, J_{\alpha}\right)$.

In general a complex hyper-Kähler manifold has no real form. A necessary condition is that the holonomy group of $\nabla^{E}$ is contained in $\operatorname{Sp}(k, l), n=k+l$, and hence preserves a quaternionic structure. Then we can define a parallel antilinear endomorphism field $j_{E}: E \rightarrow E$ such that $j_{E}^{2}=-1$ and $\omega_{E}\left(j_{E} x, j_{E} y\right)=\overline{\omega_{E}(x, y)}$ for all $x, y \in E$, where the bar denotes complex conjugation. We define a parallel antilinear endomorphism field $j_{H}: H \rightarrow H$ as the left-multiplication by the quaternion $j$ on $H=M \times \mathbb{H}$. Then $\rho=j_{H} \otimes j_{E}$ defines a field of real structures in $T M=H \otimes E$. We denote by $\mathcal{D} \subset T M$ the real eigenspace distribution of $\rho$ with eigenvalue 1 . Here $T M$ is considered as real tangent bundle of the real manifold $M$. If $M^{\rho} \subset M$ is a leaf of $\mathcal{D}$ of real dimension $4 n$ then the data $\left(g, J_{\alpha}\right)$ induce on $M^{\rho}$ a (real) hyper-Kähler structure.

4.3. Complex hyper-Kähler symmetric spaces. A complex Riemannian symmetric space is a complex Riemannian manifold $(M, g)$ such that any point is an isolated fixed point of an isometric holomorphic involution. Like in the real case one can prove that it admits a transitive complex Lie group of holomorphic isometries and that any simply connected complex Riemannian symmetric $M$ is associated to a complex symmetric decomposition

$$
\mathfrak{g}=\mathfrak{k}+\mathfrak{m}, \quad[\mathfrak{k}, \mathfrak{k}] \subset \mathfrak{k}, \quad[\mathfrak{k}, \mathfrak{m}] \subset \mathfrak{m}, \quad[\mathfrak{m}, \mathfrak{m}]=\mathfrak{k}
$$

of a complex Lie algebra $\mathfrak{g}$ together with an $\operatorname{ad}_{\mathfrak{k}}$-invariant complex scalar product on $\mathfrak{m}$. More precisely $M=G / K$, where $G$ is the simply connected complex Lie group with the Lie algebra $\mathfrak{g}$ and $K$ is the (closed) connected subgroup associated with $\mathfrak{k}$. The holonomy group of such manifold is $H=\mathrm{Ad}_{K} \mid \mathfrak{m}$. Any pseudo-Riemannian symmetric space $M=G / K$ associated with a symmetric decomposition $\mathfrak{g}=\mathfrak{k}+\mathfrak{m}$ has a canonical complexification $M^{\mathbb{C}}=G^{\mathbb{C}} / K^{\mathbb{C}}$ defined by the complexification $\mathfrak{g}^{\mathbb{C}}=$ $\mathfrak{k}^{\mathbb{C}}+\mathfrak{m}^{\mathbb{C}}$ of the symmetric decomposition. Proposition 1 remains true for complex Riemannian symmetric spaces. Ignoring the reality condition we obtain the following complex version of Theorem 1 .

THEOREM 2. Let $S \in S^{4} E, E=\mathbb{C}^{2 n}$, be a quartic polynomial invariant under all endomorphisms $S_{e, e^{\prime}} \in S^{2} E=\operatorname{sp}(E)$. Then it defines a complex hyper-Kähler symmetric space, which is associated with the following complex symmetric decomposition

$$
\mathfrak{g}=\mathfrak{h}+H \otimes E, \quad \mathfrak{h}=S_{E, E}=\operatorname{span}\left\{S_{e, e^{\prime}} \mid e, e^{\prime} \in E\right\} \subset \operatorname{sp}(E)
$$


The bracket $\wedge^{2}(H \otimes E) \rightarrow \mathfrak{h}$ is given by (3.1). The complex hyper-Kähler symmetric space $M$ associated to this symmetric decomposition is the quotient $M=M_{S}=G / K$, where $G$ is the (complex) simply connected Lie group with Lie algebra $\mathfrak{g}$ and $K \subset G$ is the connected (and closed) subgroup with Lie algebra $\mathfrak{k}=\mathfrak{h}$.

Moreover any simply connected complex hyper-Kähler symmetric space can be obtained by this construction. Two complex hyper-Kähler symmetric spaces $M_{S}$ and $M_{S^{\prime}}$ defined by quartics $S$ and $S^{\prime}$ are isomorphic if and only if $S$ and $S^{\prime}$ are in the same orbit of $\operatorname{Aut}\left(E, \omega_{E}\right)=\operatorname{Sp}(E) \cong \operatorname{Sp}(n, \mathbb{C})$.

COROLlaRY 1. There is a natural bijection between simply connected complex hyper-Kähler symmetric spaces of dimension $4 n$ up to isomorphism and $\operatorname{Sp}(n, \mathbb{C})$ orbits on the space of quartic polynomials $S \in S^{4} E$ in the symplectic vector space $E=\mathbb{C}^{2 n}$ such that

$$
S_{e, e^{\prime}} \cdot S=0 \text { for all } e, e^{\prime} \in E
$$

4.4. Classification of complex hyper-Kähler symmetric spaces. The following complex version of Proposition 4 (with similar proof) will be a crucial step in the classification of complex hyper-Kähler symmetric spaces.

Proposition 6. Let $\left(M=G / K, g, J_{\alpha}\right)$ be a simply connected complex hyperKähler symmetric space. Then the holonomy group of $M$ is solvable and $M$ admits a transitive solvable Lie group of automorphisms.

Due to Corollary 1 the classification of simply connected complex hyper-Kähler symmetric spaces reduces to the determination of quartic polynomials $S$ satisfying (4.4). Below we will determine all such polynomials. We will prove that the following example gives all such polynomials.

EXAMPLE 1: Let $E=E_{+} \oplus E_{-}$be a Lagrangian decomposition, i.e. $\omega\left(E_{ \pm}, E_{ \pm}\right)=$ 0 , of the symplectic vector space $E=\mathbb{C}^{2 n}$. Then any polynomial $S \in S^{4} E_{+} \subset S^{4} E$ satisfies the condition (4.4) and defines a simply connected complex hyper-Kähler symmetric space $M_{S}$ with Abelian holonomy algebra $\mathfrak{h}=S_{E_{+}, E_{+}} \subset S^{2} E_{+} \subset S^{2} E=$ $\operatorname{sp}(E)$.

In fact, since $E_{+}$is Lagrangian the endomorphisms from $S^{2} E_{+}$form an Abelian subalgebra of $\operatorname{sp}(E)$, which acts trivially on $E_{+}$and hence on $S^{4} E_{+}$.

THEOREM 3. Let $S \in S^{4} E$ be a quartic polynomial satisfying (4.4). Then there exists a Lagrangian decomposition $E=E_{+} \oplus E_{-}$such that $S \in S^{4} E_{+}$.

Proof: According to Theorem 2 the quartic $S$ defines a hyper-Kähler symmetric space with holonomy Lie algebra $\mathfrak{h}=S_{E, E}$. Since, by Proposition $6, \mathfrak{h}$ is solvable, Lie's theorem implies the existence of a one-dimensional $\mathfrak{h}$-invariant subspace $P=\mathbb{C} p \subset E$. There exists an $\omega$-nondegenerate subspace $W \subset E$ such that the $\omega$ orthogonal complement of $P$ is $P^{\perp}=P \oplus W$. We choose a vector $q \in E$ such that $\omega(p, q)=1$ and $\omega(W, q)=0$ and put $Q:=\mathbb{C} q$. Then we have

$$
E=P \oplus W \oplus Q
$$

Since $\mathfrak{h}$ preserves $P$ we have the following inclusion

$$
\mathfrak{h} \subset P E+W^{2}=P^{2}+P W+P Q+W^{2},
$$

where we use the notation $X Y=X \vee Y$ for the symmetric product of subspaces $X, Y \subset E$. Then the second prolongation $\mathfrak{h}^{(2)}=\left\{T \in S^{4} E \mid T_{e, e^{\prime}} \in \mathfrak{h}\right.$ for all $\left.e, e^{\prime} \in E\right\}$ 
has the following inclusion

$$
\begin{aligned}
& \mathfrak{h}^{(2)} \subset P^{3} E+P^{2} W^{2}+P W^{3}+W^{4} \\
= & P^{4}+P^{3} Q+P^{3} W+P^{2} W^{2}+P W^{3}+W^{4} .
\end{aligned}
$$

Indeed $\mathfrak{h}^{(2)} \subset \mathfrak{h}^{2}=P^{4}+P^{3} Q+P^{3} W+P^{2} Q^{2}+P^{2} W Q+P^{2} W^{2}+P Q W^{2}+P W^{3}+W^{4}$. The projection $\mathfrak{h}^{(2)} \rightarrow P^{2} Q^{2}+P^{2} W Q+P Q W^{2}$ is zero because otherwise $S_{q, q} \in \mathfrak{h} \subset$ $P E+W^{2}$ would have a nonzero projection to $Q^{2}+W Q$ or $S_{w, q} \in \mathfrak{h}$ would have a nonzero projection to $Q W$ for appropriate choice of $w \in W$. By (4.5) we can write the quartic $S$ as

$$
S=p^{3}\left(\lambda p+\mu q+w_{0}\right)+p^{2} B+p C+D
$$

where $\lambda, \mu \in \mathbb{C}, w_{0} \in W, B \in S^{2} W, C \in S^{3} W$ and $D \in S^{4} W$. From now on we will identify $S^{d} E$ with the space $\mathbb{C}\left[E^{*}\right]^{(d)}$ of homogeneous polynomials on $E^{*}$ of degree $d$. Then the $\omega$-contraction $T_{x}=\iota_{\omega x} T=T(\omega x, \ldots)$ of a tensor $T \in S^{d} E$ with a vector $x \in E$ is identified with the following homogeneous polynomial of degree $d-1$ :

$$
T_{x}=\frac{1}{d} \partial_{\omega x} T
$$

where $\partial_{\omega x} T$ is the derivative of the polynomial $T \in \mathbb{C}\left[E^{*}\right]^{(d)}$ in the direction of $\omega x=\omega(x, \cdot) \in E^{*}$. For example $p_{q}=\langle p, \omega q\rangle=\omega(q, p)=\partial_{\omega q} p=-\partial_{p^{*}} p=-1=-q_{p}$.

From $S_{p, q}=-\frac{1}{4} \mu p^{2}$ and the condition $S_{p, q} \cdot S=0$ we obtain $\mu=0$, since $p^{2} \cdot S=\mu p^{4}$. This implies $S_{p, \cdot}=0$. Next we compute:

$$
\begin{aligned}
S_{q, q} & =\frac{1}{6}\left(6 \lambda p^{2}+3 p w_{0}+B\right) \\
S_{q, w} & =-\frac{1}{12}\left(-3 p^{2} \omega\left(w_{0}, w\right)+2 p \partial_{\omega w} B+\partial_{\omega w} C\right) \\
& =-\frac{1}{12}\left(-3 p^{2} \omega\left(w_{0}, w\right)+4 p B_{w}+3 C_{w}\right) \\
S_{w, w^{\prime}} & =\frac{1}{6}\left(p^{2} B_{w, w^{\prime}}+3 p C_{w, w^{\prime}}+6 D_{w, w^{\prime}}\right)
\end{aligned}
$$

for any $w, w^{\prime} \in W$.

Now the condition (4.4) can be written as follows:

$$
\begin{aligned}
0 & =6 S_{q, q} \cdot S=\left(3 p w_{0}+B\right) \cdot S=\left(\frac{3}{2}\left(p \otimes w_{0}+w_{0} \otimes p\right)+B\right) \cdot S \\
& =\frac{3}{2}\left(2 p^{3} B_{w_{0}}+3 p^{2} C_{w_{0}}+4 p D_{w_{0}}\right)+p^{3} B w_{0}+p^{2} B \cdot B+p B \cdot C+B \cdot D \\
& =-2 p^{3} B w_{0}+\frac{9}{2} p^{2} C_{w_{0}}+p\left(6 D_{w_{0}}+B \cdot C\right)+B \cdot D .
\end{aligned}
$$

Note that $B w_{0}=-B_{w_{0}}$ and $B \cdot B=[B, B]=0$.

$$
\begin{aligned}
0= & -12 S_{q, w} \cdot S=\left(4 p B_{w}+3 C_{w}\right) \cdot S \\
= & 2\left(p^{4} \omega\left(B_{w}, w_{0}\right)+2 p^{3} B^{2} w-3 p^{2} C_{B w}-4 p D_{B w}\right) \\
& +3\left(p^{3} C_{w} w_{0}+p^{2} C_{w} \cdot B+p C_{w} \cdot C+C_{w} \cdot D\right) \\
= & 2 p^{4} \omega\left(B_{w}, w_{0}\right)+p^{3}\left(4 B^{2} w+3 C_{w} w_{0}\right)+p^{2}\left(-6 C_{B w}+3 C_{w} \cdot B\right)
\end{aligned}
$$




$$
\begin{aligned}
& +p\left(-8 D_{B w}+3 C_{w} \cdot C\right)+3 C_{w} \cdot D \\
0= & 2 S_{w, w^{\prime}} \cdot S=\frac{1}{2}\left(p \otimes C_{w, w^{\prime}}+C_{w, w^{\prime}} \otimes p\right) \cdot S+2 D_{w, w^{\prime}} \cdot S \\
= & \frac{1}{2}\left(p^{4} \omega\left(C_{w, w^{\prime}}, w_{0}\right)-2 p^{3} B C_{w, w^{\prime}}+3 p^{2} C_{C_{w, w^{\prime}}}+4 p D_{C_{w, w^{\prime}}}\right)+ \\
& 2\left(p^{3} D_{w, w^{\prime}} w_{0}+p^{2} D_{w, w^{\prime}} \cdot B+p D_{w, w^{\prime}} \cdot C+D_{w, w^{\prime}} \cdot D\right) \\
= & \frac{1}{2} p^{4} \omega\left(C_{w, w^{\prime}}, w_{0}\right)+p^{3}\left(-B C_{w, w^{\prime}}+2 D_{w, w^{\prime}} w_{0}\right)+ \\
& p^{2}\left(\frac{3}{2} C_{C_{w, w^{\prime}}}+2 D_{w, w^{\prime}} \cdot B\right)+p\left(2 D_{C_{w, w^{\prime}}}+2 D_{w, w^{\prime}} \cdot C\right)+2 D_{w, w^{\prime}} \cdot D .
\end{aligned}
$$

This gives the following system of equations:

$$
\begin{aligned}
& \text { (1) } B w_{0}=0 \\
& \text { (2) } C_{w_{0}}=0 \\
& \text { (3) } 6 D_{w_{0}}+B \cdot C=0 \\
& \text { (4) } B \cdot D=0 \\
& \text { (5) } \omega\left(B_{w}, w_{0}\right)=0 \\
& \text { (6) } 4 B^{2} w+3 C_{w} w_{0}=0 \\
& \text { (7) }-2 C_{B w}+C_{w} \cdot B=0 \\
& \text { (8) }-8 D_{B w}+3 C_{w} \cdot C=0 \\
& \text { (9) } C_{w} \cdot D=0 \\
& \text { (10) } \omega\left(C_{w} w^{\prime}, w_{0}\right)=0 \\
& \text { (11) }-B C_{w} w^{\prime}+2 D_{w, w^{\prime}} w_{0}=0 \\
& \text { (12) } \frac{3}{2} C_{C_{w} w^{\prime}}+2 D_{w, w^{\prime}} \cdot B=0 \\
& \text { (13) } D_{C_{w} w^{\prime}}+D_{w, w^{\prime}} \cdot C=0 \\
& \text { (14) } D_{w, w^{\prime}} \cdot D=0 .
\end{aligned}
$$

Note that (5) and (10) follow from (1) and (2) and that using (2) equation (6) says that the endomorphism $B$ has zero square:

$\left(6^{\prime}\right) \quad B^{2}=0$.

Eliminating $D_{w_{0}}$ in equations (3) and (11) we obtain:

(15) $0=(B \cdot C)_{w} w^{\prime}+3 B C_{w} w^{\prime}=B C_{w} w^{\prime}-C_{B w} w^{\prime}-C_{w} B w^{\prime}+3 B C_{w} w^{\prime}$

$$
=4 B C_{w} w^{\prime}-C_{B w} w^{\prime}-C_{w} B w^{\prime} .
$$

We can rewrite (7) as:

$\left(7^{\prime}\right)-2 C_{B w} w^{\prime}+C_{w} B w^{\prime}-B C_{w} w^{\prime}=0$.

Eliminating $C_{B w} w^{\prime}$ in $\left(7^{\prime}\right)$ and (15) we obtain:

(16) $-3 B C_{w} w^{\prime}+C_{w} B w^{\prime}=0$.

Since the first summand is symmetric in $w$ and $w^{\prime}$ we get

$$
C_{w} B w^{\prime}=C_{w^{\prime}} B w=C_{B w} w^{\prime} .
$$

Now using (17) we can rewrite (15) as:

(15) $2 B C_{w} w^{\prime}-C_{w} B w^{\prime}=0$. 
The equations $\left(15^{\prime}\right)$ and (16) show that $B C_{w} w^{\prime}=C_{w} B w^{\prime}=C_{B w} w^{\prime}=0$ and hence also $B \cdot C=0$. This implies $D_{w_{0}}=0$, by (3). Now we can rewrite (1-14) as:

$$
\begin{gathered}
B w_{0}=C_{w_{0}}=D_{w_{0}}=0 \\
B \cdot C=B \cdot D=0 \\
B^{2}=0 \\
C_{B w}=C_{w} B=B C_{w}=0 \\
-8 D_{B w}+3 C_{w} \cdot C=0 \\
C_{w} \cdot D=0 \\
\frac{3}{2} C_{C_{w} w^{\prime}}+2 D_{w, w^{\prime}} \cdot B=0 \\
D_{C_{w} w^{\prime}}+D_{w, w^{\prime}} \cdot C=0 \\
D_{w, w^{\prime}} \cdot D=0 .
\end{gathered}
$$

Now to proceed further we decompose $K:=\operatorname{ker} B=W_{0} \oplus W^{\prime}$, where $W_{0}=\operatorname{ker} \omega \mid K$ and $W^{\prime}$ is a (nondegenerate) complement. Let us denote by $W_{1}$ a complement to $K$ in $W$ such that $\omega\left(W^{\prime}, W_{1}\right)=0$. Then $W_{0}+W_{1}$ is the $\omega$-orthogonal complement to the $B$-invariant nondegenerate subspace $W^{\prime}$. This shows that $B W_{1} \subset\left(W_{0}+W_{1}\right) \cap K=$ $W_{0}$. Moreover since $W_{1} \cap K=0$ the map $B: W_{1} \rightarrow W_{0}$ is injective and hence $\operatorname{dim} W_{1} \leq \operatorname{dim} W_{0}$. On the other hand $\operatorname{dim} W_{1} \geq \operatorname{dim} W_{0}$, since $W_{0}$ is an isotropic subspace of the symplectic vector space $W_{0}+W_{1}$. This shows that $B: W_{1} \rightarrow W_{0}$ is an isomorphism.

Lemma 3. $C \in S^{3} K$ and $D \in S^{4} K$.

Proof: Since $W_{0}=B W$ the equation (4.9) shows that $C_{W_{0}}=0$, which proves the first statement. From (4.10) and the identity

$$
\left(C_{x} \cdot C\right)_{y}=\left[C_{x}, C_{y}\right]-C_{C_{x} y}
$$

we obtain

$$
D_{B x, y}+D_{B y, x}=\frac{3}{8}\left(\left(C_{x} \cdot C\right)_{y}+\left(C_{y} \cdot C\right)_{x}\right)=-\frac{3}{4} C_{C_{x} y} .
$$

The equation $B \cdot D=0$ (4.7) reads:

$$
0=(B \cdot D)_{x, y}=\left[B, D_{x, y}\right]-D_{B x, y}-D_{x, B y} .
$$

Using this (4.12) yields:

$$
D_{B x, y}+D_{B y, x}=\left[B, D_{x, y}\right]=-D_{x, y} \cdot B=\frac{3}{4} C_{C_{x} y} .
$$


Now from (4.16) and (4.17) we obtain that

$$
0=C_{C_{x} y} z=C_{z} C_{x} y
$$

for all $x, y, z \in W$. This implies $\left[C_{x}, C_{y}\right]=0$ for all $x, y \in W$ and hence

$$
C_{x} \cdot C=0
$$

for all $x \in W$, by (4.15). Finally this shows that $D_{W_{0}}=0$ by (4.10). This proves the second statement.

Lemma 4. $D_{x, y} C_{z}=C_{z} D_{x, y}=0$ for all $x, y, z \in W$.

Proof: Using (4.13) we compute:

$$
D_{x, y} C_{z} w=D_{C_{z} w, x} y=-\left(D_{z, w} \cdot C\right)_{x} y=-\left(\left[D_{z, w}, C_{x}\right] y-C_{D_{z, w} x} y\right) .
$$

From (4.11) we get:

$$
\begin{aligned}
0= & \left(C_{x} \cdot D\right)_{z, w} y=\left[C_{x}, D_{z, w}\right] y-D_{C_{x} z, w} y-D_{z, C_{x} w} y \\
& =C_{x} D_{z, w} y-D_{z, w} C_{x} y-D_{y, w} C_{x} z-D_{z, y} C_{x} w
\end{aligned}
$$

and hence:

$$
\left[D_{z, w}, C_{x}\right] y=-D_{y, w} C_{x} z-D_{z, y} C_{x} w
$$

and

$$
C_{D_{z, w} x} y=C_{y} D_{z, w} x=D_{z, w} C_{x} y+D_{x, w} C_{y} z+D_{z, x} C_{y} w .
$$

Now we eliminate the $C D$-terms from (4.19) arriving at:

$$
D_{x, y} C_{z} w=\left(D_{y, w} C_{x} z+D_{z, y} C_{x} w+D_{z, w} C_{x} y+D_{x, w} C_{y} z+D_{z, x} C_{y} w\right)
$$

Considering all the permutations of $(x, y, z, w)$ we get 6 homogeneous linear equations for the 6 terms of equation (4.20) with the matrix:

$$
\left(\begin{array}{cccccc}
-1 & 1 & 1 & 1 & 1 & 1 \\
1 & -1 & 1 & 1 & 1 & 1 \\
1 & 1 & -1 & 1 & 1 & 1 \\
1 & 1 & 1 & -1 & 1 & 1 \\
1 & 1 & 1 & 1 & -1 & 1 \\
1 & 1 & 1 & 1 & 1 & -1
\end{array}\right)
$$

This is the matrix of the endomorphism $-2 \mathrm{Id}+e \otimes e$ in the arithmetic space $\mathbb{R}^{6}$, where $e=e_{1}+\ldots+e_{6} ;\left(e_{i}\right)$ the standard basis. It has eigenvalues $(4,-2,-2,-2,-2,-2)$. This shows that the matrix is nondegenerate and proves the lemma.

For a symmetric tensor $T \in S^{d} W$ we denote by

$$
\Sigma_{T}:=\operatorname{span}\left\{T_{x_{1}, x_{2}, \ldots, x_{d-2}} x_{d-1} \mid x_{1}, x_{2}, \ldots x_{d-1} \in W\right\} \subset W
$$

the support of $T$. 
Lemma 5. The supports of the tensors $B \in S^{2} W, C \in S^{3} W$ and $D \in S^{4} W$ admit the following inclusions

$$
\Sigma_{B}+\Sigma_{C} \subset \operatorname{ker} B \cap \operatorname{ker} C \cap \operatorname{ker} D, \quad \Sigma_{D} \subset \operatorname{ker} B \cap \operatorname{ker} C .
$$

Moreover $\Sigma_{B}+\Sigma_{C}$ is isotropic and $\omega\left(\Sigma_{D}, \Sigma_{B}+\Sigma_{C}\right)=0$.

Proof: The first statement follows from $B^{2}=B C_{x}=B D_{x, y}=C_{x} B=C_{x} C_{y}=$ $C_{x} D_{y, z}=D_{x, y} B=D_{x, y} C_{z}=0$ for all $x, y, z \in W$. The second statement follows from the first and the definition of support, e.g. if $z=C_{x} y \in \Sigma_{C}$ and $w \in \Sigma_{B}+\Sigma_{C}+\Sigma_{D} \subset$ $\operatorname{ker} C$ we compute:

$$
\omega(z, w)=\omega\left(C_{x} y, w\right)=-\omega\left(y, C_{x} w\right)=0
$$

Lemma 6. The Lie algebra $D_{W, W} \subset S^{2} W \cong \operatorname{sp}(W)$ is solvable.

Proof: This follows from Proposition 6, since $D \in S^{4} W$ satisfies (4.4) and hence defines a complex hyper-Kähler symmetric space with holonomy Lie algebra $D_{W, W}$. It also follows from the solvability of $S_{E, E}$ as we show now. In terms of the decomposition $E=P+W+Q$ an endomorphism

$S_{x, y}=\left(\lambda p^{4}+p^{3} w_{0}+p^{2} B+p C+D\right)_{x, y}=\left(p^{2} B+p C+D\right)_{x, y}=B(x, y) p^{2}-p C_{x} y+D_{x, y}$,

where $x, y \in W$, is represented by

$$
\left(\begin{array}{ccc}
0 & -\left(C_{x} y\right)^{t} & B(x, y) \\
0 & D_{x, y} & -C_{x} y \\
0 & 0 & 0
\end{array}\right) .
$$

Since the Lie algebra $S_{E, E}$ is solvable this implies that the Lie algebra $D_{W, W}$, which corresponds to the induced representation of $S_{E, E}$ on $P^{\perp} / P \cong W$, is also solvable.

LEMMA 7.

$$
\omega\left(w_{0}, \Sigma_{B}+\Sigma_{C}+\Sigma_{D}\right)=0
$$

Proof: Note that $w_{0} \in \operatorname{ker} B \cap \operatorname{ker} C \cap \operatorname{ker} D$, due to equations (1-3) and (4.7). This implies the lemma. In fact, if e.g. $y=B x \in \Sigma_{B}$ then

$$
\omega\left(w_{0}, y\right)=\omega\left(w_{0}, B x\right)=-\omega\left(B w_{0}, x\right)=0,
$$

which shows that $\omega\left(w_{0}, \Sigma_{B}\right)=0 \square$

Now to finish the proof of Theorem 3 we will use induction on the dimension $\operatorname{dim} E=2 n$. If $n=1$ the (solvable) holonomy algebra $\mathfrak{h}$ is a proper subalgebra of $S^{2} E \cong \operatorname{sl}(2, \mathbb{C})$. Without loss of generality we may assume that either

a) $\mathfrak{h}=\mathbb{C} p^{2}$ or

b) $\mathfrak{h}=\mathbb{C} p q$ or

c) $\mathfrak{h}=\mathbb{C} p^{2}+\mathbb{C} p q$

where $(p, q)$ is a symplectic basis of $E$. In the all three cases the Lie algebra $S_{E, E}=$ $\mathfrak{h} \subset \mathbb{C} p^{2}+\mathbb{C} p q$ and hence

$$
S=\lambda p^{4}+\mu p^{3} q
$$


In the cases b) and c) we have that $p q \in \mathfrak{h}$ and since

$$
p q \cdot S=\frac{1}{2}(p \otimes q+q \otimes p) \cdot S=-2 \lambda p^{4}-\mu p^{3} q=0
$$

it follows that $S=0$. In the case a) from $S_{E, E}=\mathfrak{h}=\mathbb{C} p^{2}$ we have that $S=\lambda p^{4}$. This tensor is invariant under $\mathfrak{h}=\mathbb{C} p^{2}$ and belongs to the fourth symmetric power of the Lagrangian subspace $\mathbb{C} p \subset E$. This establishes the first step of the induction. Now by induction using equation (4.14) and Lemma 6 we may assume that $\Sigma_{D}$ is isotropic. Now Lemma 5 and Lemma 7 show that $\mathbb{C} w_{0}+\Sigma_{B}+\Sigma_{C}+\Sigma_{D}$ is isotropic and hence is contained in some Lagrangian subspace $E_{+} \subset E$. This implies that $S \in S^{4} E_{+}$.

Now we give a necessary and sufficient condition for a symmetric manifold $M=$ $M_{S}, S \in S^{4} E_{+}$, to have no flat de Rham factor.

Proposition 7. The complex hyper-Kähler symmetric space $M_{S}, S \in S^{4} E_{+}$, has no flat de Rham factor if and only if the support $\Sigma_{S}=E_{+}$.

Proof: If $M=M_{S}=G / K$ has a flat factor $M_{0}$, such that $M=M_{1} \times M_{0}$, then this induces a decomposition $E=E^{1} \oplus E^{0}$ and $S \in S^{4} E_{1}$; hence $\Sigma_{S} \subset E_{1} \cap E_{+} \neq E_{+}$. Conversely let $S \in S^{4} E_{+}$, assume that $E_{+}^{1}=\Sigma_{S} \subset E_{+}$is a proper subspace and choose a complementary subspace $E_{+}^{0}$. We denote by $E_{-}^{1}$ and $E_{-}^{0}$ the annihilator of $\omega E_{+}^{0}$ and $\omega E_{+}^{1}$ respectively. Let us denote $E^{1}=E_{+}^{1} \oplus E_{-}^{1}, E^{0}=E_{+}^{0} \oplus E_{-}^{0}, \mathfrak{m}^{1}=$ $H \otimes E^{1}$ and $\mathfrak{m}^{0}=H \otimes E^{0}$. Then $E^{0}, E^{1} \subset E$ are $\omega$-nondegenerate complementary subspaces and $\mathfrak{m}^{0}, \mathfrak{m}^{1} \subset \mathfrak{m}=T_{o} M$ are $g$-nondegenerate complementary subspaces. Since $S \in S^{4} E_{+}^{1}$ the Lie algebra $\mathfrak{g}=\mathfrak{h}+\mathfrak{m}=\left(\mathfrak{h}+\mathfrak{m}^{1}\right) \oplus \mathfrak{m}^{0}$ has the Abelian direct summand $\mathfrak{m}^{0}$, see (3.1), which gives rise to a flat factor $M^{0} \subset M_{S}=M^{1} \times M^{0}$.

THEOREM 4. Any simply connected complex hyper-Kähler symmetric space without flat de Rham factor is isomorphic to a complex hyper-Kähler symmetric space of the form $M_{S}$, where $S \in S^{4} E_{+}$and $E_{+} \subset E$ is a Lagrangian subspace of the complex symplectic vector space $E=\mathbb{C}^{2 n}$. Moreover there is a natural 1-1 correspondence between simply connected complex hyper-Kähler symmetric spaces without flat factor up to isomorphism and orbits $\mathcal{O}$ of the group $\operatorname{Aut}\left(E, \omega, E_{+}\right) \mid E_{+}=\left\{A \in S p(E) \mid A E_{+}=\right.$ $\left.E_{+}\right\} \mid E_{+} \cong \mathrm{GL}\left(E_{+}\right) \cong \mathrm{GL}(n, \mathbb{C})$ on the space $S^{4} E_{+}$such that $\Sigma_{S}=E_{+}$for all $S \in \mathcal{O}$.

Proof: This is a corollary of Theorem 2, Theorem 3 and Proposition 7.

Let $M=G / K$ be a simply connected complex hyper-Kähler symmetric space without flat factor. By Theorem 3 and Proposition 7 it is associated to quartic polynomial $S \in S^{4} E_{+}$with support $\Sigma_{S}=E_{+}$. Now we describe the Lie algebra aut $\left(M_{S}\right)$ of the full group of automorphisms, i.e. isometries which preserve the hypercomplex structure, of $M_{S}$.

Theorem 5. Let $M_{S}=G / K$ be as above. Then the full automorphism algebra is given by

$$
\operatorname{aut}\left(M_{S}\right)=\operatorname{aut}(S)+\mathfrak{g},
$$

where $A \in \operatorname{aut}(S)=\left\{B \in \operatorname{gl}\left(E_{+}\right) \mid B \cdot S=0\right\}$ acts on $\mathfrak{g}=\mathfrak{h}+\mathfrak{m}$ as follows. It preserves the decomposition and acts on $\mathfrak{h}=S_{E, E}$ by

$$
\left[A, S_{x, y}\right]=S_{A x, y}+S_{x, A y}
$$

for all $x, y \in E$ and on $\mathfrak{m}=H \otimes E$ by

$$
[A, h \otimes e]=h \otimes A e,
$$


where $\mathrm{gl}\left(E_{+}\right)$is canonically embedded into $\operatorname{sp}(E)$.

Proof: By (the complex version of) Proposition 1 it is sufficient to determine the centralizer $\mathfrak{c}$ of $\operatorname{sp}(1, \mathbb{C})$ in the full isotropy algebra $\tilde{\mathfrak{h}}=\operatorname{aut}(R) \supset \operatorname{sp}(1, \mathbb{C}) \oplus \mathfrak{h}$. Equation (2.2) shows that

$$
\mathfrak{c}=\{\operatorname{Id} \otimes A \mid A \in \operatorname{sp}(E), A \cdot S=[A, S(\cdot, \cdot)]-S(A \cdot, \cdot)-S(\cdot, A \cdot)=0\} .
$$

From $A \cdot S=0$ we obtain that the commutator $\left[A, S_{x, y}\right]=S_{A x, y}+S_{x, A y}$ for all $x, y \in E$ and $A \Sigma_{S}=A E_{+} \subset E_{+}$. This implies $\mathfrak{c}=\operatorname{aut}(S)$.

5. Classification of Hyper-Kähler Symmetric Spaces. Using the description of complex hyper-Kähler symmetric spaces given in Theorem 4 we will now classify (real) hyper-Kähler symmetric spaces. Recall that a simply connected pseudoRiemannian manifold is called indecomposable if it is not a Riemannian product of two pseudo-Riemannian manifolds. Any simply connected pseudo-Riemannian manifold can be decomposed into the Riemannian product of indecomposable pseudoRiemannian manifolds. By Wu's theorem [W] a simply connected pseudo-Riemannian manifold is indecomposable if and only if its holonomy group is weakly irreducible, i.e. has no invariant proper nondegenerate subspaces. Therefore it is sufficient to classify (real) hyper-Kähler symmetric spaces with indecomposable holonomy.

Let $\left(M=G / K, g, J_{\alpha}\right)$ be a hyper-Kähler symmetric space associated to a symmetric decomposition (2.1). The complexified tangent space of $M$ is identified with $\mathfrak{m}^{\mathbb{C}}=H \otimes E$, the tensor product of to complex symplectic vector spaces with quaternionic structure $j_{H}$ and $j_{E}$ such that $\rho=j_{H} \otimes j_{E}$ is the complex conjugation of $\mathfrak{m}^{\mathbb{C}}$ with respect to $\mathfrak{m}$. By Theorem 1 it is defined by a quartic polynomial $S \in S^{4} E$ satisfying the conditions of the theorem. Moreover the holonomy algebra $\mathfrak{h}$ acts trivially on $H$ and is identified with the real form of the complex Lie algebra $S_{E, E} \subset \operatorname{sp}(E)$ given by $\mathfrak{h}=\operatorname{span}\left\{S_{j e, e^{\prime}}-S_{e, j e^{\prime}} \mid e, e^{\prime} \in E\right\}=\left\{A \in S_{E, E} \mid\left[A, j_{E}\right]=0\right\} \subset \operatorname{sp}(E)^{j_{E}}$.

The quartic polynomial $S$ defines also a complex hyper-Kähler symmetric space $M^{\mathbb{C}}=G^{\mathbb{C}} / K^{\mathbb{C}}$, which is the complexification of $M=G / K$. By Theorem $3, S \in S^{4} L$ for some Lagrangian subspace $L \subset E$. Recall that the symplectic form $\omega=\omega_{E}$ together with the quaternionic structure $j=j_{E}$ define a Hermitian metric $\gamma=\gamma_{E}=$ $\omega_{E}\left(\cdot, j_{E} \cdot\right)$ of (real) signature $(4 k, 4 l), n=k+l$, which coincides with the signature of the pseudo-Riemannian metric $g$ (we normalize $\gamma_{H}=\omega_{H}\left(\cdot, j_{H} \cdot\right.$ ) to be positive definite). We may decompose $\gamma$-orthogonally $L=L^{0} \oplus L^{+} \oplus L^{-}$, such that $\gamma$ vanishes on $L^{0}$ is positive definite on $L^{+}$and negative definite on $L^{-}$.

LEMMA 8.

(i) $j L^{0}=L^{0}$ and

(ii) $L^{+}+L^{-}+j L^{+}+j L^{-} \subset E$ is an $\omega$-nondegenerate and $\gamma$-nondegenerate $\mathfrak{h}$ invariant subspace (with trivial action of $\mathfrak{h}$ ).

Proof: We show first that $L+j L^{0}$ is $\omega$-isotropic and hence $L+j L^{0}=L$ since $L$ is Lagrangian. Indeed $L \supset L^{0}$ is $\omega$-isotropic and also $j L^{0}$ because $\omega$ is $j$-invariant. So it suffices to remark that $\omega\left(L, j L^{0}\right)=0$ :

$$
\omega\left(L, j L^{0}\right)=\gamma\left(L, L^{0}\right)=0 .
$$

This implies that $j L^{0} \subset L$. Since $\gamma\left(L, j L^{0}\right)=-\omega\left(L, L^{0}\right)=0$, we conclude that $j L^{0} \subset \operatorname{ker} \gamma \mid L=L^{0}$. This proves (i).

To prove (ii) it is sufficient to check that the subspace $L^{+}+L^{-}+j L^{+}+j L^{-} \subset E$ is nondegenerate with respect to $\gamma$, since it is $j$-invariant. First we remark that $\gamma$ is 
positive definite on $L^{+}$and $j L^{+}$and negative definite on $L^{-}$and $j L^{-}$, due to the $j$-invariance of $\gamma: \gamma(j x, j x)=\gamma(x, x), x \in E$. So to prove (ii) it is sufficient to check that $j L^{+} \oplus j L^{-}$is $\gamma$-orthogonal to the $\gamma$-nondegenerate vector space $L^{+}+L^{-}$:

$$
\gamma\left(L^{+}+L^{-}, j L^{+}+j L^{-}\right)=\omega\left(L^{+}+L^{-}, L^{+}+L^{-}\right)=0 .
$$

By Theorem 1 the quartic polynomial $S$ must satisfy the reality condition $\left[S_{j e, e^{\prime}}-S_{e, j e^{\prime}}, j\right]=0$. Now we describe all such polynomials.

The quaternionic structure $j$ on $E$ is compatible with $\omega$, i.e. $\omega(j x, j y)=\overline{\omega(x, y)}$ for all $x, y \in E$ and it induces a real structure (i.e. an antilinear involution) $\tau:=$ $j \otimes j \otimes \cdots \otimes j$ on all even powers $S^{2 r} E \subset E \otimes E \otimes \cdots \otimes E$. For $S \in S^{2 r} E$ and $x_{1}, \cdots, x_{2 r} \in E$ we have

$$
(\tau S)\left(x_{1}, \cdots, x_{2 r}\right)=\overline{S\left(j x_{1}, \cdots, j x_{2 r}\right)} .
$$

Note that the fixed point set $\operatorname{sp}(E)^{\tau}=\{A \in \operatorname{sp}(E) \mid[A, j]=0\} \cong \operatorname{sp}(k, l)$.

Proposition 8. Let $(E, \omega, j)$ be a complex symplectic vector space with a quaternionic structure $j$ such that $\omega(j x, j y)=\overline{\omega(x, y)}$ for all $x, y \in E$. Then a quartic polynomial $S \in S^{4} E$ satisfies the reality condition $\left[S_{j e, e^{\prime}}-S_{e, j e^{\prime}}, j\right]=0$ if and only if $S \in\left(S^{4} E\right)^{\tau}=\operatorname{span}\left\{T+\tau T \mid T \in S^{4} E\right\}$.

Proof: The reality condition for $S \in S^{4} E$ can be written as

$$
\left[S_{j x, j y}+S_{x, y}, j\right] z=0
$$

for all $x, y, z \in E$. Contracting this vector equation with $j w \in E$ by means of $\omega$ and using the compatibility between $j$ and $\omega$ we obtain the equivalent condition

$$
\begin{aligned}
0 & =-\omega\left(j w,\left[S_{j x, j y}+S_{x, y}, j\right] z\right) \\
& =S(j x, j y, j z, j w)-\overline{S(j x, j y, z, w)}+S(x, y, j z, j w)-\overline{S(x, y, z, w)} .
\end{aligned}
$$

Now putting $x=y=z=w=u$ we obtain:

$$
0=S(j u, j u, j u, j u)-\overline{S(j u, j u, u, u)}+S(u, u, j u, j u)-\overline{S(u, u, u, u)}
$$

and putting $x=i u$ and $y=z=w=u$ we obtain:

$$
0=-i S(j u, j u, j u, j u)-i \overline{S(j u, j u, u, u)}+i S(u, u, j u, j u)+i \overline{S(u, u, u, u)} .
$$

Comparing these two equations we get $S(j u, j u, j u j, u)=\overline{S(u, u, u, u)}$, i.e. $S=\tau S$. This shows that the reality condition implies that $S \in\left(S^{4} E\right)^{\tau}$. Conversely the condition $S=\tau S$ can be written as

$$
S(j x, j y, j z, j w)=\overline{S(x, y, z, w)} \text { for all } x, y, z, w \in E .
$$

Changing $z \rightarrow j z$ and $w \rightarrow j w$ in this equation we obtain

$$
S(j x, j y, z, w)=\overline{S(x, y, j z, j w)} \text { for all } x, y, z, w \in E .
$$

These two equations imply (5.1) and hence the reality condition.

Now we are ready to classify simply connected hyper-Kähler symmetric spaces. We will show that the following construction gives all such symmetric spaces. 
Let $(E, \omega, j)$ be a complex symplectic vector space of dimension $2 n$ with a quaternionic structure $j$ such that $\omega(j x, j y)=\overline{\omega(x, y)}$ for all $x, y \in E$ and $E=E_{+} \oplus E_{-}$a $j$-invariant Lagrangian decomposition. Such a decomposition exists if and only if the Hermitian form $\gamma=\omega(\cdot, j \cdot)$ has real signature $(4 m, 4 m)$, where $\operatorname{dim}_{\mathbb{C}} E=2 n=4 m$. Then any polynomial $S \in\left(S^{4} E_{+}\right)^{\tau}=S^{4} E_{+} \cap\left(S^{4} E\right)^{\tau}$ satisfies the condition (4.4) and the reality condition, by Proposition 8 . Hence by Theorem 1 it defines a (real) simply connected hyper-Kähler symmetric space $M_{S}$ with Abelian holonomy algebra $\mathfrak{h}=$ $\left(S_{E_{+}, E_{+}}\right)^{\tau}=S_{E_{+}, E_{+}} \cap\left(S^{2} E\right)^{\tau}=\operatorname{span}\left\{S_{j e, e^{\prime}}-S_{e, j e^{\prime}} \mid e, e^{\prime} \in E\right\} \subset \operatorname{sp}(E)^{\tau} \cong \operatorname{sp}(m, m)$.

THEOREM 6. Any simply connected hyper-Kähler symmetric space without flat de Rham factor is isomorphic to a hyper-Kähler symmetric space of the form $M_{S}$, where $S=T+\tau T, T \in S^{4} E_{+}$and $E_{+} \subset E$ is a $j$-invariant Lagrangian subspace of the complex symplectic vector space $E$ with compatible quaternionic structure $j$. A hyper-Kähler symmetric space of the form $M_{S}$ has no flat factor if and only if it complexification has no flat factor, which happens if and only if the support $\Sigma_{S}=E_{+}$. Moreover there is a natural 1-1 correspondence between simply connected hyper-Kähler symmetric spaces without flat factor up to isomorphism and orbits $\mathcal{O}$ of the group $\operatorname{Aut}\left(E, \omega, j, E_{+}\right)\left|E_{+}=\left\{A \in S p(E) \mid[A, j]=0, A E_{+}=E_{+}\right\}\right| E_{+} \cong \mathrm{GL}(m, \mathbb{H})$ on the space $\left(S^{4} E_{+}\right)^{\tau}$ such that $\Sigma_{S}=E_{+}$for all $S \in \mathcal{O}$.

Proof: Let $M$ be a simply connected hyper-Kähler symmetric space. We first assume that it is indecomposable. Then the holonomy algebra $\mathfrak{h}$ is weakly irreducible. By Theorem 1, $M=M_{S}$ for some quartic polynomial $S \in S^{4} E$ satisfying (4.4) and the reality condition (3.2). By Proposition 8 the reality condition means that $S \in\left(S^{4} E\right)^{\tau}$. On the other hand, by Theorem $3 S \in S^{4} L$ for some Lagrangian subspace $L$ of $E$. Now the weak irreducibility of $\mathfrak{h}$ and Lemma 8 imply that $L=L^{0}$ is $j$-invariant. This proves that $S \in\left(S^{4} E_{+}\right)^{\tau}$, where $E_{+}=L=L^{0}$ is a $j$-invariant Lagrangian subspace of $E$. This shows that $M$ is obtained from the above construction. Any simply connected hyper-Kähler symmetric space $M$ without flat factor is the Riemannian product of indecomposable ones, say $M=M_{1} \times M_{2} \times \cdots \times M_{r}$, and we may assume that $M_{i}=M_{S_{i}}, S_{i} \in S^{4} E_{i}$. Therefore $M$ is associated to the quartic polynomial $S=S_{1} \oplus S_{2} \oplus \cdots \oplus S_{r} \in S^{4} E, E=E_{1} \oplus E_{2} \oplus \cdots \oplus E_{r}$. Moreover $S$ satisfies (4.4) and (3.2) if the $S_{i}$ satisfy (4.4) and (3.2). This shows that any simply connected hyper-Kähler symmetric space is obtained from the above construction.

It is clear that the complexification $M_{S}^{\mathbb{C}}$ has a flat factor if $M_{S}$ has a flat factor. Conversely let us assume that $M_{S}^{\mathbb{C}}$ has a flat factor, hence $\Sigma_{S} \subset E_{+}$is a proper subspace. Since $j \Sigma_{S}=j S_{E, E} E=S_{E, E} j E=S_{E, E} E=\Sigma_{S}$ there exists a $j$-invariant complementary subspace $E_{+}^{\prime}$ in $E_{+}$. Denote by $E_{-}^{\prime}$ the annihilator of $\Sigma_{S}$ in $E_{-}$then $E^{\prime}=E_{+}^{\prime} \oplus E_{-}^{\prime}$ is an $\omega$-nondegenerate and $j$-invariant subspace of $E$ on which the holonomy $\mathfrak{h}^{\mathbb{C}}=S_{E, E} \subset S^{2} \Sigma_{S}$ acts trivially. Then the corresponding real subspace $\left(H \otimes E^{\prime}\right)^{\rho} \subset \mathfrak{m}=(H \otimes E)^{\rho}$ is a $g$-nondegenerate subspace on which the holonomy $\mathfrak{h}$ acts trivially. By Wu's theorem [W] it defines a flat de Rham factor.

Now the last statement follows from the corresponding statement in Theorem 1. $\square$

COROLlaRY 2. Any hyper-Kähler symmetric space without flat factor has signature $(4 m, 4 m)$. In particular its dimension is divisible by 8 .

Corollary 3. Let $M=M_{S}$ be a complex hyper-Kähler symmetric space without flat factor associated with a quartic $S \in S^{4} E_{+}$, where $E_{+} \subset E$ is a Lagrangian subspace. It admits a real form if and only if there exists a quaternionic structure $j$ 
on $E$ compatible with $\omega$ preserving $E_{+}$such that $\tau S=S$, where $\tau$ is the real structure on $S^{4} E$ induced by $j$. In particular $\operatorname{dim}_{\mathbb{C}} M$ has to be divisible by 8 .

Let $M=G / K$ be a simply connected hyper-Kähler symmetric space without flat factor. By Theorem 6 it is associated to a quartic polynomial $S \in\left(S^{4} E_{+}\right)^{\tau}$ with support $\Sigma_{S}=E_{+}$. Now we describe the Lie algebra aut $\left(M_{S}\right)$ of the full group of automorphisms, i.e. isometries which preserve the hypercomplex structure of $M_{S}$.

THEOREM 7. Let $M_{S}=G / K$ be as above. Then the full automorphism algebra is given by

$$
\operatorname{aut}\left(M_{S}\right)=\operatorname{aut}(S)+\mathfrak{g}
$$

where $\operatorname{aut}(S)=\left\{A \in \operatorname{gl}\left(E_{+}\right) \mid[A, j]=0, A \cdot S=0\right\}$ acts on

$\mathfrak{g}=\mathfrak{h}+\mathfrak{m}, \mathfrak{h}=\left\{A \in S_{E, E} \mid[A, j]=0\right\}=\operatorname{span}\left\{S_{j x, y}-S_{x, j y} \mid x, y \in E\right\}, \mathfrak{m}=(H \otimes E)^{\rho}$

as in Theorem 5.

Proof: The proof is similar to that of Theorem 5.

6. Low Dimensional Hyper-Kähler Symmetric Spaces.

6.1 Complex hyper-Kähler symmetric spaces of dimension $\leq 8$.

\section{Dimension 4}

Assume that $M$ is a simply connected complex hyper-Kähler symmetric space of dimension 4. Applying Theorem 4 we conclude that $M=M_{S}$ for some $S \in S^{4} E_{+}$, where $E_{+} \subset E$ is a one-dimensional subspace $E_{+}=\mathbb{C} e$. This proves:

THEOREM 8. There exists up to isomorphism only one non-flat simply connected complex hyper-Kähler symmetric space of dimension 4: $M=M_{S}$ associated with the quartic $S=e^{4}$.

\section{Dimension 8}

Any eight-dimensional simply connected complex hyper-Kähler symmetric space is associated with a quartic $S \in S^{4} E_{+}$, where $E_{+} \subset E$ is a Lagrangian subspace of $E=\mathbb{C}^{4}$. We denote by $\left(e, e^{\prime}\right)$ a basis of $E_{+}$.

THEOREM 9. Eight-dimensional simply connected complex hyper-Kähler symmetric space are in natural $1-1$ correspondence with the orbits of the group $\mathrm{CO}(3, \mathbb{C})=$ $\mathbb{C}^{*} \cdot \mathrm{SO}(3, \mathbb{C})$ on the space $S_{0}^{2} \mathbb{C}^{3}$ of traceless symmetric matrices. The complex hyperKähler symmetric space associated with a traceless symmetric matrix $A$ is the manifold $M_{S(A)}$, where $S(A) \in S^{4} \mathbb{C}^{2}$ is the quartic polynomial which corresponds to $A$ under the $\mathrm{SO}(3, \mathbb{C})$-equivariant isomorphism $S_{0}^{2} \mathbb{C}^{3} \cong S_{0}^{2} \wedge^{2} \mathbb{C}^{3} \cong S_{0}^{2} S^{2} \mathbb{C}^{2}=S^{4} \mathbb{C}^{2}$.

The classification of $\mathrm{SO}(3, \mathbb{C})$-orbits on $S_{0}^{2} \mathbb{C}^{3}$ was given by Petrov $[\mathrm{P}]$ in his classification of Weyl tensors of Lorentzian 4-manifolds.

Proof: By Theorem 4 the classification of eight-dimensional simply connected complex hyper-Kähler symmetric spaces reduces to the description of orbits of the group $\mathrm{GL}\left(E_{+}\right)=\mathrm{GL}(2, \mathbb{C})$ on $S^{4} \mathbb{C}^{2} \subset S^{2} S^{2} \mathbb{C}^{2}$. Fixing a volume form $\sigma$ on $\mathbb{C}^{2}$ we can identify $S^{2} \mathbb{C}^{2}$ with $\operatorname{sp}(1, \mathbb{C}) \cong \operatorname{so}(3, \mathbb{C})$. Then the Killing form $B$ is an $\operatorname{SL}(2, \mathbb{C})$ invariant and we have the $\mathrm{GL}(2, \mathbb{C})$-invariant decomposition: $S^{2} S^{2} \mathbb{C}^{2}=S_{0}^{2} S^{2} \mathbb{C}^{2} \oplus$ $\mathbb{C} B$. The action of $\mathrm{SL}(2, \mathbb{C})$ on $S^{2} \mathbb{C}^{2}$ is effectively equivalent to the adjoint action of $\mathrm{SO}(3, \mathbb{C})$. The problem thus reduces essentially to the determination of the orbits of $\mathrm{SO}(3, \mathbb{C})$ on $S_{0}^{2} \mathbb{C}^{3}$. 
5.1. Hyper-Kähler symmetric spaces of dimension $\leq 8$. By Corollary 3 the minimal dimension of non-flat hyper-Kähler symmetric spaces is 8 .

THEOREM 10. Eight-dimensional simply connected hyper-Kähler symmetric space are in natural 1-1 correspondence with the orbits of the group $\mathbb{R}^{+} \cdot \mathrm{SO}(3)$ on the space $S_{0}^{2} \mathbb{R}^{3}$ of traceless symmetric matrices. The hyper-Kähler symmetric space associated with a traceless symmetric matrix $A$ is the manifold $M_{S(A)}$, where $S(A) \in\left(S^{4} \mathbb{C}^{2}\right)^{\tau}$ is the quartic polynomial which corresponds to $A$ under the $\mathrm{SO}(3)$-equivariant isomorphism $S_{0}^{2} \mathbb{R}^{3} \cong\left(S^{4} \mathbb{C}^{2}\right)^{\tau}$.

\section{REFERENCES}

[BD] M.L. BARBeris, I. DotTi-Miatello, Hypercomplex structures on a class of solvable Lie groups, Quart. J. Math. Oxford II. Ser., 47 (1996), no. 188, pp. 389-404.

[B1] M. BERGER, Classification des espaces homogènes symétriques irréductibles, C.R. Acad. Sci. Paris, 240 (1955), pp. 2370-2372.

[B2] M. BERGER, Structure et classification des espaces homogènes symétriques à groupe d'isométries semi-simple, C. R. Acad. Sci. Paris, 241 (1955), pp. 1696-1698.

[Bo] M. Bordemann, Nondegenerate invariant bilinear forms on nonassociative algebras, Acta Math. Univ. Comen., New Ser. 66 (1997), no. 2, pp. 151-201.

[CP] M. CAHEN, M. PARKer, Sur des classes d'espaces pseudo-riemanniens symétriques, Bull. Soc. Math. Bely., 22 (1970), pp. 339-354.

[CW] M. CAhen, N. Wallach, Lorentzian symmetric spaces, Bull. Amer. Math. Soc., 76 (1970), pp. 585-591.

[F] A.S. FEDEnKo, Spaces defined by Lie group endomorphisms ( $\Phi$-spaces), Collection of articles dedicated to the memory of German Fedorovič Laptev, Trudy Geometr. Sem., 4 (1973), pp. 231-267.

[J] D. JoYce, Compact hypercomplex and quaternionic manifolds, J. Diff. Geom., 35 (1992), pp. $743-761$.

[K] V.G. KAC, Infinite dimensional Lie algebras, Cambridge University Press, 1990.

[MR1] A. MEdina, P. Revoy, Algèbres de Lie et produit scalaire invariant, Ann. Sci. Ec. Norm. Super. IV. Ser., 18 (1985), pp. 553-561.

[MR2] A. MEdinA, P. Revoy, Algèbres de Lie orthogonales. Modules orthogonaux, Commun. Algebra, 21 (1993), no.7, pp. 2295-2315.

[O-V] A.L. ONishchiK, E.B. Vinberg (EDS.), Lie groups and Lie algebras III, Encyclodaedia of Mathematical Sciences, 41, Springer, 1994.

[P] A.Z. Petrov, Einstein spaces, Pergamon, Oxford, 1969.

[SSTVP] Ph. Spindel, A. Sevrin, W. Troost, A. Van Proeyen, Extended super-symmetric $\sigma$-models on group manifolds, Nuclear Phys. B, 308 (1988), pp. 662-698.

[W] H. Wu, On the de Rham decomposition theorem, Illinois J. Math., 8 (1964), pp. 291-311. 